\title{
Multiobjective Optimization Based-Approach for Discovering Novel Cancer Therapies
}

\author{
Arthur W. Mahoney, Gregory J. Podgorski, and Nicholas S. Flann
}

\begin{abstract}
Solid tumors must recruit new blood vessels for growth and maintenance. Discovering drugs that block tumor-induced development of new blood vessels (angiogenesis) is an important approach in cancer treatment. The complexity of angiogenesis presents both challenges and opportunities for cancer therapies. Intuitive approaches, such as blocking VegF activity, have yielded important therapies. But there maybe opportunities to alter nonintuitive targets either alone or in combination. This paper describes the development of a high-fidelity simulation of angiogenesis and uses this as the basis for a parallel search-based approach for the discovery of novel potential cancer treatments that inhibit blood vessel growth. Discovering new therapies is viewed as a multiobjective combinatorial optimization over two competing objectives: minimizing the estimated cost of practically developing the intervention while minimizing the simulated oxygen provided to the tumor by angiogenesis. Results show the effectiveness of the search process by finding interventions that are currently in use, and more interestingly, discovering potential new approaches that are nonintuitive yet effective.
\end{abstract}

Index Terms-Cancer therapy, cellular Potts model, CPM, Glazier-Graner-Hogeweg model, GGH, multiobjective optimization, parallel search, computational discovery, angiogenesis, VegF.

\section{INTRODUCTION}

ANCER is to be the leading cause of death throughout the world in 2010 [9]. By 2020, between 20 to 26 million people are predicted to be diagnosed with cancer and 13 to 17 million people will die of cancer. With estimated five year survival rates only 65 percent [34], there is an urgent need for new, more effective cancer therapies.

There is an expanding pipeline of anticancer drugs and therapies [62]. The first critical phase of this treatment pipeline is the discovery of therapies that slow the growth of an avascular tumor by stopping, slowing, or remodeling its vascularization. Searching for new treatments in the laboratory is exceedingly difficult not only because of the inherent complexity of cancer, but also because of time and expense. These methods must first implement carefully planned changes in an animal model system, and then observe the resulting effects such as inhibiting vascularization or tumor growth [54]. These time-consuming procedures are a principal factor limiting the rate of new treatment discovery.

Computational and mathematical models for cancer study speed the exploration of possible cancer therapies and open new possibilities for treatments and their potential has been long recognized [10]. Computational models have helped to understand specific processes of cancer such as tumor growth [23], metastasis [61], and angiogenesis [40],

- A.W. Mahoney and N.S. Flann are with Computer Science Department, Utah State University, Logan, UT 84321-4205.

E-mail: art.mahoney@utah.edu, nick.flann@usu.edu.

- G.J. Podgorski is with Biology Department and Center for Integrated BioSystems, Utah State University, Logan, UT 84321.

E-mail: greg.podgorski@usu.edu.

Manuscript received 7 Oct. 2009; revised 3 Feb. 2010; accepted 30 Mar. 2010; published online 7 May 2010.

For information on obtaining reprints of this article, please send e-mail to: tcbb@computer.org, and reference IEEECS Log Number TCBB-2009-10-0179. Digital Object Identifier no. 10.1109/TCBB.2010.39.
[52], and have enabled evaluation and improvement of therapies "in silico" [45], [4]. Computational models provide many advantages over laboratory-based methods: they run orders of magnitude faster and can simulate days of cancer development in seconds; they can evaluate a treatment by measuring tumor growth or computing the oxygen delivery efficiency of a newly formed vascular system [2]; and they can easily implement alternative therapies, chemotherapy schedules, and doses through parameter/behavior-rule changes [11], [21], [32]. Computational models of cancer development offer the potential to significantly improve the efficiency of finding new therapies.

Tumors grow and spread by first recruiting new blood vessels from the host tissues in a process called angiogenesis. This process is initiated when cells in the center of the microtumor experience hypoxia and secret vascular endothelial growth factor (VegF). Existing host vessels respond by sprouting a network of blood vessels toward the tumor to provide the needed nutrition and oxygen. Antiangiogenic drugs increase survival rates among cancer patients by disrupting or modifying this tumor-induced angiogenesis [12], [53], [18]. Evidence suggests that there are a variety of ways these drugs work. Some antiangiogenic treatments (e.g., the VEGF-blocking antibody bevacizumab) repair the tumor-induced vessels - which tend to be poorly structured and leaky - and then apply conventional chemotherapy or radiation therapy. In this approach, the repaired vessels increase survival by enabling more efficient delivery of chemotherapeutics and increased oxygen for effective radiation therapy [33]. Other approaches seek to prevent the initiation of vascularization and the subsequent formation of functional vasculature for the tumor [44] These approaches starve a microtumor at a very early stage, arrest its growth, and reduce the effect of hypoxia and the resultant secretion of VegF. The cumulative outcome is to 
halt the progression from the initial microtumor to a growing, vascularized tumor.

This paper seeks to identify new potential therapies that block the initial development of the vascular network. Future work will consider the alternative approach, where vessels are repaired and optimized for oxygen delivery. A novel and general-purpose computational discovery system is introduced that rapidly generates thousands of potential therapies, then evaluates their effectiveness through the use of a cell-based simulation model. The best interventions discovered are pooled and reported.

Using the computational modeling work of [7] and [2] as a springboard, this work presents a high-fidelity simulation model of the initial stages of angiogenesis and integrates multiple spatial scales at the subcellular, cellular, and tissue levels. This simulation system is sufficiently abstract to be computationally feasible, yet is sufficiently detailed to identify specific medically relevant intervention targets represented as the model parameters. The model simulates the first steps of angiogenesis, when new blood vessels grow toward the tumor, form loops that allow blood to flow (anastomosis), and secrete oxygen and nutrients to support the tumor. The discovery algorithm evaluates potential interventions by measuring the amount of simulated oxygen provided to the tumor during the first 72 hours of angiogenesis. The computational search-based approach has the potential to suggest promising treatments to laboratory-based researchers.

\section{BACKGROUND}

\subsection{Angiogenesis Blocking Treatments}

The state of the art in angiogenesis blocking treatments is reviewed in [56], [44], and [53]. Successful treatments discovered so far target single-component mechanisms that directly influence the development of vessels. One important example is blocking the activity of $\mathrm{VegF}$, a protein secreted by hypoxic cells that induces the proliferation of new blood vessels. Existing treatments either bind to VegF to interfere with binding to its receptors, or bind to VegF receptors on the endothelial cells, preventing activation and growth [17]. Another set of drugs affects how proteolytic enzymes interact with the extracellular matrix [57], and other treatments slow the growth of both the endothelial and tumor cells directly [27], or disrupt endothelial cell signalling networks [19].

To discover alternative therapies, this work considers disrupting other component mechanisms of angiogenesis, either singularly or in groups, that may effect the morphology and resulting efficiency of the new blood vessel network. Potential targets for intervention include cell-specific adhesion or cell elasticity, haptotaxis, and filopodia flexibility or extension rates. Importantly, novel therapies maybe discovered by considering combinations of targets. The complexity of angiogenesis presents many component mechanisms that could be disrupted in multiple ways, requiring the search of a large combinatorial space of possible therapies that is infeasible using laboratory-based biological methods. By utilizing a highfidelity simulation of angiogenesis and parallel resources, this large combinatorial space can be efficiently searched and new potential therapies discovered. A further advantage of this computational approach is the lack of human bias in the search process making discovery of nonintuitive, currently unavailable, but potentially effective cancer treatments possible.

\subsection{Treatment Discovery as Optimization}

The high-fidelity simulation of angiogenesis used in this research takes as input a vector of numerical parameters that each describe a component mechanism of angiogenesis. When provided as input to the angiogenesis model, each vector of parameters will increase, decrease, or leave unchanged the simulated oxygen supplied to the tumor. The discovery of effective therapies maybe viewed as a combinatorial optimization problem over the space of parameter vectors where the estimated cost (i.e., difficulty) of clinical implementation and the simulated oxygen supplied to the tumor are two distinct objective functions, which often conflict. A good potential therapy will minimize both the estimated cost and the oxygen supply to the tumor simultaneously.

In multiobjective optimization, a set of best solutions exist, each of which maybe considered optimal according to the specifications of the given problem. To understand this optimal set, a dominance relation between solutions is defined as a partial ordering over the set of all solutions, where solution $a$ dominates solution $b$ if all of the objectives of $a$ are strictly better than those of solution $b$ [20]. The optimal solution set is called the Pareto optimal frontier [26] and comprises of those solutions that are not dominated by any other solution.

Multiobjective optimization, then, is the search for solutions that lie on the Pareto optimal frontier. There are many computational search-based techniques that are used for multiobjective optimization. Computational approaches are preferred over mathematical programming techniques because they are not affected by continuity or the concavity of the Pareto optimal front, they don't require derivatives, and they tend to converge rapidly to near-Pareto optimal solutions [59]. Two common computational search-based techniques are evolutionary algorithms and simulated annealing.

Evolutionary algorithms are well suited for multiobjective optimization due to their ability to simultaneously search a population of multiple Pareto optimal solutions and their ability to cover large expanses of space [16]. A significant amount of research has been performed on developing fitness assignment strategies that yield good convergence [16], [64], [65]. The fitness of a solution $a$, is measured by the number of solutions it dominates, how many solutions dominate $a$, how deeply $a$ dominates the rest of the population, or a combination of all three [65]. Most evolutionary algorithms used for multiobjective optimization also incorporate techniques to maintain diversity along the Pareto optimal frontier and to prevent loss of good solutions using either elitism or a form of solution archiving [16]. However, standard evolutionary algorithms impose no limits on the complexity of the solutions found, and are known to produce complex, 
bloated solutions [38] that could not be implemented as medical treatments.

Simulated annealing algorithms have also been used with some success for solving multiobjective optimization problems [59]. These techniques have become popular due to their simplicity and ability to rapidly generate a set of Pareto optimal solutions with relatively low computational cost. Like the evolutionary algorithm approach to multiobjective optimization, many variants have been developed and used successfully to solve real-world problems [6], [15], [60]. Generally, the variants differ with respect to their acceptance criterion, whether or not a potential solution is classified as an improvement over previous solutions, and their management of the Pareto optimal solution set.

\subsection{Overview}

The rest of this paper is organized as follows: First, the Monte Carlo-based search engine is described in Section 3, followed by a description of our modified Glazier-GranerHogeweg angiogenesis simulation system in Section 3.3. Section 4 describes potentially effective cancer therapies discovered by the search engine. Finally, the findings of this work are discussed in Section 5.

\section{Search Method}

Each potential therapy is represented as a vector of 40 parameter deviations, $\Delta \vec{P}$, from the vector of normative parameter values, $\overrightarrow{P_{N}}$, such that $\vec{P}=\overrightarrow{P_{N}}+\Delta \vec{P}$. Each parameter encodes a qualitative behavior of a component mechanism included in the angiogenesis model such as endothelial cell growth, cell adhesion, the forces of chemotaxis and haptotaxis, ECM adhesion and degradation, filopodia extension and lifetime, or VegF secretion from the tumor, diffusion through the host tissue, and uptake by endothelial cells. The normative parameter vector represents the default, untreated condition and a vector of deviations represents a potential treatment. The normative values for each of the 40 parameters have been defined according to the literature and simulation studies of standard blood vessel morphologies [3], [7], [8]. Along with a normative value, each parameter is assigned a range of possible values according to what changes are physically valid in the angiogenesis model and maybe medically feasible to change now or in the near future. Each mechanism and its associated parameters are described in Section 3.3. The normative values and ranges of the 40 parameters used in this simulation are given in Tables 2 and 3.

Multiobjective optimization occurs in the parameter deviation space, defined as a subset of the $\mathbb{R}^{40}$ vector space, where each $\Delta \vec{P} \in$ contains the 40 parameter deviations (dimensions) whose values are bounded above and below according to their respective, predefined ranges.

The cost objective function, which estimates the medical cost of implementing the effects described by a deviation vector, is defined as $\operatorname{Cost}(\Delta \vec{P})$, and described in detail in Section 3.2.1. The true cost of actually implementing a parameter deviation as an effective and safe therapy is very difficult to determine due to the need to operationalize each parameter change into a clinical intervention. The oxygen objective function, defined by $O_{2}(\Delta \vec{P})$, is used to evaluate how much oxygen is supplied to a tumor given the effects induced by a vector of parameter deviations, $\Delta \vec{P}$. The oxygen supplied to the tumor is obtained by running the angiogenesis model that is described in detail in Section 3.3.

The combinatorial search problem for this application is to identify a set of Pareto optimal interventions that minimize both the estimated cost of the intervention and the oxygen provided to the tumor at the initial phase of vessel growth. The search algorithm approach needs to comprehensively explore and discover solutions that are novel and minimal. Novel solutions exploit neglected mechanisms or unexpected interactions among the many mechanisms in the model. Minimal solutions achieve the best oxygen reduction with the lowest number of parameters changed, combined with the smallest deviation of those parameters. A comprehensive search will consider many distinct solutions with no initial bias in what combinations of parameters are considered.

To satisfy these requirements, an unbiased Monte Carlo approach is used to generate initial solutions as random deviations to random combinations of parameters. To achieve the requirement of minimality, promising solutions are reduced by systematic elimination and minimization of parameter deviations until a local minimum is found. All solutions are organized into a near-Pareto optimal frontier so as to retain promising solutions while rejecting inferior solutions. To ensure practicality of discovered solutions, the search engine begins with solutions that contain at most three parameter changes from nominal. A local beam search algorithm combined with hill climbing algorithm is used to organize the reduction and minimization of solutions.

The method applied here is top-down, in that the search moves from more complex solutions to simpler solutions, terminating with locally minimal solutions. More efficient bottom-up approaches have been applied to some multiplecriteria problems [1], [13] but since they require at least weak monotonicity in all the objective functions, they cannot be applied to this problem. In particular, the oxygen fitness of a solution emerges from a complex interaction of mechanisms that have no direct or ordered relationship to the extent and scope of the parameter deviations.

Throughout the course of the search, $O_{2}(\Delta \vec{P})$ is called repeatedly to evaluate a potential solution over a range of random initial conditions. Each simulation of angiogenesis needed to compute $O_{2}(\Delta \vec{P})$ can take upwards of 5 to 7 minutes on a modern processor. Because many thousands of these are performed, an effective combinatorial search cannot be feasibly executed without the use of parallel computation. Fortunately, individual executions of the angiogenesis simulation share no information, therefore they are independent and can be performed simultaneously. This form of parallel approach is known as an "embarrassingly parallel" method. Embarrassingly parallel algorithms are capable of achieving optimal (linear) speedup so long as each processor is always working at its maximum capacity. Although the speedup achieved and scalability of our method is not the focus of our study, by using simple parallelism on computational resources provided by the US National Science Foundation (NSF) Terra Grid and the Arctic Region Supercomputing Center, we were able to rapidly search through potential solutions by running between 512 and 2,048 $\mathrm{O}_{2}$ objective function evaluations simultaneously. 


\subsection{Improving Local Beam Search}

Let $S$ be a population of $n$ parameter deviation vectors sampled from For $1 \leq j \leq n$, each deviation vector $\Delta \vec{P}_{j} \in S$ is denoted by $\Delta \vec{P}_{j}=\left(\delta p_{1}, \ldots, \delta p_{i}, \ldots, \delta p_{40}\right)$, where $\delta p_{i}$ is the deviation from the nominal value of the ith parameter. If the deviation $\delta p_{i} \neq 0$ then the ith parameter is said to be "active." Let $A(\Delta \vec{P})$ denotes the set of active parameters in the deviation vector $\Delta \vec{P}$.

The improving local beam search takes as input the maximum number of active (nonzero) parameter deviations, $k$, and an initial population of random deviation vectors, $S_{k}$, then searches through the set of vectors, $\Delta \vec{P} \in X$, where $1 \leq$ $|A(\Delta \vec{P})| \leq k$ for vectors that minimize the cost and oxygen absorbed by the tumor. The initial population $S_{k}$ is seeded using Random Sample $(k, n)$ that uniformly samples the set of deviation vectors in with exactly $k$ active parameters. The search engine operates by systematically reducing the number of active parameters $i$ in each population $S_{i}$ at each iteration until the vectors in the current population have only one parameter remaining. During the search, the best solutions discovered so far are archived in the set $L^{*}$, which is returned by the search method.

The search algorithm proceeds in an iterative fashion alternatively generating random mutations of the deviation vectors in the current population and selecting the most promising to be mutated and subsequently carry over to the population in the next iteration. The algorithm is described in pseudocode in Algorithm 1 . At the $i$ th iteration, the estimated cost and the amount of oxygen supplied to the tumor are first evaluated and stored for each $\Delta \vec{P} \in S_{i}$ using $\operatorname{Cost}()$ and $O_{2}()$. The best deviation vectors in $S_{i}$ are then selected using $\varepsilon$ ParetoOptimal $\left(S_{i}\right)$ that returns a subset of $S_{i}$ that lie on or near the Pareto optimal frontier of the current population, $S_{i}$. To determine those solutions that are near the Pareto optimal frontier, $\varepsilon$ ParetoOptimal $\left(S_{i}\right)$ returns only those solutions that are dominated by at most $\varepsilon$ other solutions in the population. The best-so-far solution archive, $L^{*}$, is then updated with these near-optimal solutions from $S_{i}$.

Algorithm 1. ImprovingLocalBeamSearch $(k, n)$ returns a set, $L^{*}$, containing all the near-Pareto optimal solution vectors found during the discovery search.
1: ImprovingLocalBeamSearch $(k, n)$
2: $L^{*} \leftarrow \emptyset$
3: $S_{k} \leftarrow$ RandomSample $(k, n)$
4: for $i=k$ downto 1 do
5: for each $\Delta \vec{P}_{j} \in S_{i}$
6: $\quad \Delta \vec{P}_{j}$. cost $\leftarrow \operatorname{Cost}\left(\Delta \vec{P}_{j}\right)$
7: $\quad \Delta \vec{P}_{j} . o_{2} \leftarrow O_{2}\left(\Delta \vec{P}_{j}\right)$
8: end for
9: $\quad S_{i} \leftarrow \varepsilon$ ParetoOptimal $\left(S_{i}\right)$
10: $\quad L^{*} \leftarrow \varepsilon$ ParetoOptimal $\left(S_{i} \cup L^{*}\right)$
11: $\quad S_{i-1} \leftarrow$ ReduceResample $\left(S_{i}, n\right)$
12: end for
13: return $L^{*}$

The next population of deviation vectors, $S_{i-1}$, is generated by the ReduceResample $\left(S_{i}, n\right)$ function, which returns a new set of $n$ vectors by simplifying and mutating each $\Delta \vec{P} \in S_{i}$ on the near-Pareto optimal frontier. The ReduceResample function produces simplified children from each deviation vector, $\Delta \vec{P}$ in $S_{i}$, by iterating $j$ through
$\Delta \vec{P}^{\prime}$ s active parameters $A(\Delta \vec{P})$ and placing a copy of $\Delta \vec{P}$ in $S_{i-1}$ with the $j$ th parameter set inactive and the remaining parameters unchanged. A parameter of a deviation vector is set inactive by setting its deviation to 0 . The remaining slots in the population are generated in ReduceResample by randomly selecting solutions $\Delta \vec{P}$ from the set of simplified solutions, and then randomly resampling each active parameter $\delta p_{j} \in A(\Delta \vec{P})$. The resample operator picks a new random value for parameter $\delta p_{j}$ uniformly from its range constraints. The population $S_{i-1}$ for the next iteration of the local beam search is composed of the simplified nonmutated and simplified mutated children of the nearPareto optimal population $S_{i}$. The algorithm terminates when $k=1$ since the $k=0$ case is the no treatment case.

\subsection{Improving Hill Climber}

Once the improving local beam search algorithm is completed, the hill climber is applied to each of the potential deviation vectors in $L^{*}$ to ensure that all solutions found are locally minimal. The hill climber is an iterative local search method that takes as input a deviation vector and incrementally adjusts its parameters to reduce $\operatorname{Cost}()$ as well as $\mathrm{O}_{2}()$. At each iteration of the local search, the hill climber only considers the vectors neighboring the current deviation vector, $\Delta \vec{P}$, that are orthogonal to $\Delta \vec{P}$ and nearby $\Delta \vec{P}$ in that their deviation values have been reduced geometrically by $\gamma$ (set at a fixed step size of 0.9 ). The hill climber selects the neighbor $\Delta \vec{P}_{j}$ that dominates $\Delta \vec{P}$ and it not dominated by any other neighbor to become the current vector of the next iteration. If none of the neighbor deviation vectors are selected, then $\Delta \vec{P}$ is returned by the hill climber and the solution is considered locally optimized. Algorithm 2 describes the hill climbing optimization algorithm in pseudocode where OrthogonalNeighbors $(\Delta \vec{P}, \Delta d)$ return the set of $\Delta \vec{P}_{j}$, where $1 \leq j \leq k$ and $k$ is the number of active parameters. The function, MinimumNeighbor $(N, \Delta \vec{P})$, evaluates the neighbors $N$ using $O_{2}(\Delta \vec{P})$ and returns the selected $\Delta \vec{P}_{j}$.

Algorithm 2. The improving hill climber optimization algorithm minimizes an input deviation vector, $\Delta \vec{P}_{i n}$, by monotonically reducing the estimated cost and the simulated oxygen supplied to the tumor.

ImprovingHillClimber $\left(\Delta \vec{P}_{i n}, \gamma\right)$ returns a minimized deviation vector whose cost cannot be further decreased or the oxygen supplied to the tumor cannot be reduced. The $\gamma$ represents the geometric step size.

$$
\begin{aligned}
& \text { 1: ImprovingHillClimber }\left(\Delta \vec{P}_{i n}, \gamma\right) \\
& \text { 2: } \Delta \vec{P} \leftarrow \Delta \vec{P}_{\text {in }} \\
& \text { 3: while true do } \\
& \text { 4: } \quad \Delta \vec{P}_{\min } \leftarrow \Delta \vec{P} \\
& \text { 5: } \quad \quad \text { OrthogonalNeighbors }(\Delta \vec{P}, \gamma) \\
& \text { 6: } \quad \Delta \vec{P}_{\min } \leftarrow \text { MinimumNeighbor }(N, \Delta \vec{P}) \\
& \text { 7: } \quad \text { if } \Delta \vec{P}_{\min }=\Delta \vec{P} \text { then } \\
& \text { 8: } \quad \text { return } \Delta \vec{P}_{\text {min }} \\
& \text { 9: else } \\
& \text { 10: } \quad \Delta \vec{P} \leftarrow \Delta \vec{P}_{\min } \\
& \text { 11: end if } \\
& \text { 12: end while }
\end{aligned}
$$




\subsubsection{Evaluating the Cost Objective Function}

Given a deviation vector defined as

$\Delta \vec{P}=\left(\delta p_{1}, \ldots, \delta p_{i}, \ldots, \delta p_{40}\right)$. Let the range of parameter $i$ be $R_{i}=H_{i}-L_{i}$ (where $H_{i}$ and $L_{i}$ are the allowable high and low parameter value, respectively, given in Tables 2 and 3 , then the cost of a deviation vector is estimated by summing the weighted individual deviations from nominal:

$$
\begin{gathered}
\operatorname{Cost}(\Delta \vec{P})=\sum_{1}^{40} \omega_{i} *\left(\frac{\delta p_{i}}{R_{i}}\right)^{2}, \\
\omega_{i}= \begin{cases}2.0, & \text { if cost of } p_{i} \text { is low, } \\
4.0, & \text { if cost of } p_{i} \text { is medium, } \\
8.0, & \text { if cost of } p_{i} \text { is high. }\end{cases}
\end{gathered}
$$

The relative costs of changing parameters $\omega_{i}$, given in Tables 2 and 3, are determined by reviewing the literature on known medical intervention strategies [56] and considering the complexity of the biological mechanism and subsystem to be manipulated [25], [37]. The medical cost is estimated based on whether specific treatments are currently available (low cost), have the potential to become available in the foreseeable future (medium cost), or are predicted to be difficult to develop with current technologies (high cost). The cost function could add a factor that increased the cost based on $k$ (the number of parameter deviations in the solution) but this was not included since $k$ never exceeded three and the summation captures a linear growth in cost as $k$ grows. In fact, the cost function could be replaced by one that nonlinearly combines multiple factors such as risk to patient, manufacturing cost, development cost, etc., since the algorithm does not exploit any structure of the cost function and treats it as a "black box."

\subsubsection{Evaluation of Oxygen Objective Function}

The oxygen supplied to the tumor is evaluated with the angiogenesis tissue simulation system implemented as an extension of CompuCell [31]. To evaluate a solution, the simulation takes the $\Delta \vec{P}$ vector of parameter values as input and initializes the parameters for each mechanism using the normative values and the individual $\delta p_{i} \in \Delta \vec{P}$. The random seed used to establish the initial configuration of the stroma is set and the simulation started. As the simulation runs, new blood vessels sprout and form loops, blood starts to flow and oxygen is secreted and delivered to the tumor. The $\mathrm{O}_{2}$ score over the course of the simulation run is calculated by accumulating the $\mathrm{O}_{2}$ absorbed by the tumor every 12 hours of simulated time. Because of the stochastic nature of the simulation, the model produces distinct morphologies when using different random seeds, even when initialized using identical $\Delta \vec{P}$ s. For this reason, the oxygen objective function, $\mathrm{O}_{2}(\Delta \vec{P})$ is taken as average $\mathrm{O}_{2}$ score over 32 parameter initializations using different random seeds and subsequent runs of the angiogenesis model.

\subsection{Angiogenesis Model}

The angiogenesis computational model combines and extends the work of Merks et al. [47], Owen et al. [11], and Bauer et al. [7] to simulate the first three days of angiogenesis over a tissue region $368 \mu \mathrm{m}$ wide and $165 \mu \mathrm{m}$ deep. The model is detailed enough to account for the shape of individual endothelial cells and their extension and movement over space and time. Critically, sprout formation and anastomosis are not modeled explicitly, but emerge from the independent behavior of each cell over the tissue domain.

The simulation integrates:

1. a Glazier-Graner-Hogeweg(GGH) (also known as the cellular Potts model or CPM) that represents the tissue as a discrete spatial domain and captures mechanisms of endothelial cell growth, adhesion, tip cell chemotaxis, haptotaxis [31] and filopodia growth and exploration [58], and stromal cells and extracellular matrix (ECM) fiber adhesion and degradation;

2. a continuous model of VegF secretion from the tumor, diffusion through the stroma, activation of endothelial cells, and uptake;

3. a network flow model that estimates blood flow through the irregular network of vessels that emerge during angiogenesis; and

4. a continuous model of oxygen secretion from vessel loops, diffusion through the stroma and uptake by the tumor.

This model produces behavior such as vessel branching, loop formation (anastomosis), progression and termination of tip movement, basement membrane degradation, and activation and growth of endothelial cells to form new vessels. Significantly, all these complex behaviors emerge from interactions among the simpler, biologically relevant and cell-based component mechanisms of the model.

\subsubsection{Initial Conditions}

The simulation of angiogenesis begins with an existing blood vessel already activated by VegF secreted from a nearby avascular tumor. Although there are multiple forms of VegF as well as additional secreted growth factors such as angiopeitin that cooperate in angiogenesis, the model makes the simplification of grouping these into a single diffusible growth factor, VegF. For computational simplicity, the tissue domain is modeled in only two dimensions along the plane of the existing vessel (as in [49] and [2], illustrated in Fig. 1a). The existing vasculature is along the top border and is represented as a collection of endothelial cells enclosed by a basal lamina of ECM. In Fig. 1a, the activated endothelial cells of the vessel have entered the cell cycle and begun to increase in size and divide. The activated cell closest to the tumor and there receiving the highest levels of VegF differentiates into a tip cell. Tip cells form a regular spaced pattern, and once differentiated, explore the stroma and lead the underlying endothelial cell stalk. In contrast to stalk cells, tip cells do not grow or divide [28], [30]. The tumor lies along the bottom and is represented as a thin layer of cells. These cells represent only the outermost layer of a larger solid tumor. The distance from the existing vasculature to the surface of the tumor wall is $165 \mu \mathrm{m}$, and represents the limits of oxygen diffusion from existing vessels [35], [42]. The complexity of the stroma is represented as in [3], [8] across the area between the tumor and the vasculature explicitly as an isotropic arrangement of medium (white), ECM fibers (yellow) and an assortment of stromal cells (blue). 


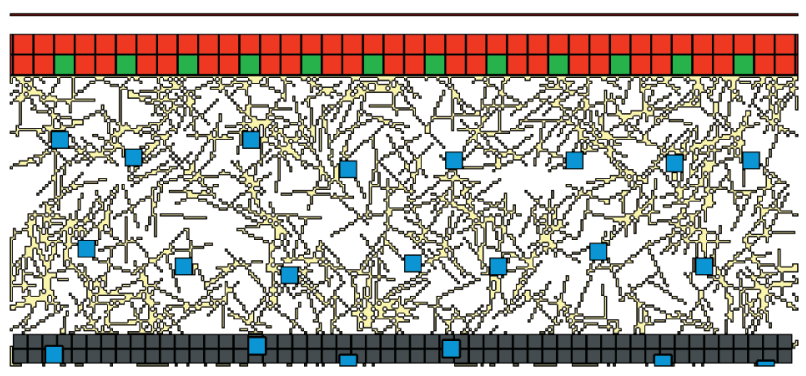

(a)

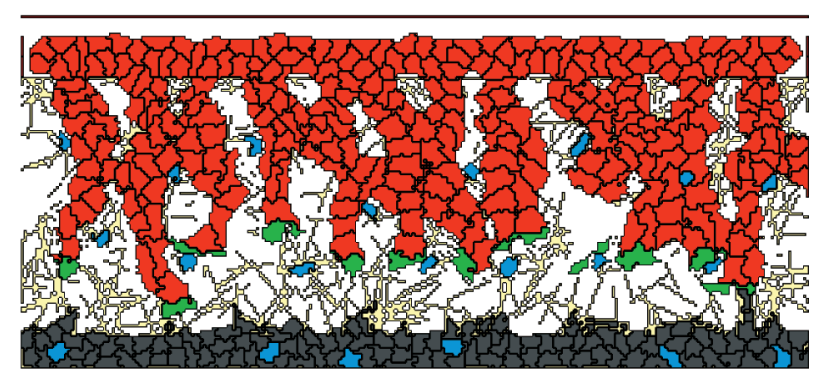

(b)

Fig. 1. (a) The initial cell type domain conditions of the angiogenesis simulation. The colors used are given in Table 1. The initial vessel is along the top, with the tumor surface along the bottom. The initial vessel is enclosed by a basement membrane of ECM (seen as thin yellow horizontal line separating the vessel from the stroma). (b) A typical vessel morphology produced by the simulation system after 600 Monte Carlo steps (MCS) or 30 hours of simulated vessel development time. In all the simulations reported here, the tissue array is 268 by 120 pixels. (a) Initial conditions. (b) Typical morphology.

\subsubsection{Glazier-Graner-Hogeweg Tissue Model}

The GGH represents the tissue domain as a cellular automata-a discrete two-dimensional array of regular "automaton," referred to as lattice sites in the GGH literature [29]. An individual cell or stromal structure is represented as a contiguous region of lattice sites of the same identity. Cell-cell and cell-ECM contacts occur through adjacent lattice sites that belong to different cells or cells and the ECM. As the simulation is run, lattice sites stochastically change to the identity of neighboring sites (referred to as a flip), causing cells to form new contacts, change shape and move. The GGH provides a uniform and principled method of specifying cellular constraints (such as a cell must be contiguous) and mechanisms (such as differential adhesion between cells) by modeling development as a stochastic search for a minimum energy configuration of the lattice site domain. Each constraint or mechanism is specified independently as a contribution to the calculation of total energy of a given configuration. See [47] for more details of the GGH model.

In our system, additional continuous layers are added to the discrete GGH model to represent cell-stroma interactions, cell-signaling events that are important in angiogenesis and $\mathrm{O}_{2}$ diffusion. The cell-signalling layer represents the concentration of VegF over the tissue as a 2D-array of the same spatial resolution as the GGH [7]. VegF is a key signaling molecule secreted from the tumor cells that drives the initiation and subsequent recruitment of endothelial cells. Although there are multiple forms of $\mathrm{VegF}$ and other secreted signaling molecules such as angiopoietins that play important roles in angiogenesis [51], these are not explicitly considered in the model. Another layer represents the distribution of ECM fibers in the underlying tissue as a 2D-array of the same spatial resolution as the GGH. Endothelial cell-stroma interactions significantly contribute to the fidelity and realism of the model since the ECM fibers are known to bend the vessel sprouts and cause them to form loops, allowing blood flow and increasing oxygen and nutrient delivery [7]. The $O_{2}$ layer will be discussed later in Section 3.3.

To run the simulation forward from the initial conditions, brief updates for each array (GGH tissue array, continuous ECM, and continuous $\mathrm{VegF}$ ) are interlaced with advection affects ignored. To update the GGH tissue array, one Monte Carlo Step (an MCS or $268 \times 120$ pixel flip attempts, see [41] for more details) is executed. One MCS simulates approximately three minutes of real time. To update the ECM and $\mathrm{VegF}$ arrays, the concentrations of ECM and VegF are modified based on endothelial cell interactions described below. A typical vessel morphology is given in Fig. $1 \mathrm{~b}$ following 600 MCS or 30 hours of simulated development time.

The following sections give a brief description of each mechanism in the angiogenesis model, its implementation in the GGH and its control parameters that are included in the $\Delta \vec{P}$ and listed in Tables 2 and 3.

\subsubsection{Cell Contiguity, Area, and Shape}

In most types of morphogenesis, cells actively adjust their shape [63]. In angiogenesis, the stalk cells elongate and form single or double files of cells which mature into new vessels [5], and the tip cells stretch and extend filopodia [22]. In this work, the shape of each cell is controlled by constraints on a cell's area and its isoperimetric quotient (or IPQ, which is the ratio of the area to the perimeter squared, normalized so that the IPQ of a circle is 1.0). Cells with a low IPQ form tight circles and pack densely; high IPQ cells can elongate or extend filopodia. The GGH implements each constraint as part of its energy minimization procedure by introducing terms that contribute energy to the score of a tissue configuration as shape constraints are violated. A cell's energy contribution increases as its shape parameters (its area and IPQ) move away from their given target values (given in Table 2). The relative contribution of each

TABLE 1

Types Used in Simulation

\begin{tabular}{|l|l|l|}
\hline \multicolumn{3}{|c|}{ Cell Types } \\
\hline Symbol & Description & Color in Figures \\
\hline$\tau_{m}$ & Medium & White \\
$\tau_{t u}$ & Tumor & Black \\
$\tau_{s}$ & Endothelial Stalk & Red \\
$\tau_{t}$ & Endothelial Tip & Green \\
$\tau_{s t}$ & Stromal Cell & Blue \\
$\tau_{e}$ & ECM Fiber & Yellow \\
\hline
\end{tabular}


TABLE 2

Parameters 1 through 15 Governing the Model

\begin{tabular}{|r|l|r|r|r|r|l|}
\hline \multicolumn{7}{|c|}{ Mechanism: Cell size and shape } \\
\hline$i$ & Param. & $N_{i}$ & $L_{i}$ & $H_{i}$ & Cost & Description \\
\hline 1 & $A_{t}$ & 45 & 35 & 60 & high & Endothelial tip $\tau_{t}$ target area \\
2 & $\lambda_{A_{t}}$ & 8.0 & 0.5 & 14.0 & medium & Endothelial tip $\tau_{t}$ area energy weight \\
3 & $\phi_{t}$ & 7.0 & 2.0 & 12.0 & high & Endothelial tip $\tau_{t}$ target isoperimetric quotient \\
4 & $\lambda_{\phi_{t}}$ & 3.0 & 0.5 & 14.0 & medium & Endothelial tip $\tau_{t}$ IPQ energy weight \\
\cline { 2 - 7 } 5 & $A_{s}$ & 45.0 & 35 & 60 & high & Endothelial stalk $\tau_{s}$ target area \\
6 & $\lambda_{A_{s}}$ & 3.0 & 0.5 & 14.0 & medium & Endothelial stalk $\tau_{s}$ area energy weight \\
7 & $\phi_{s}$ & 6.0 & 2.0 & 12.0 & high & Endothelial stalk $\tau_{s}$ target isoperimetric quotient \\
8 & $\lambda_{\phi_{s}}$ & 1.0 & 0.5 & 14.0 & medium & Endothelial stalk $\tau_{s}$ IPQ energy weight \\
\cline { 2 - 7 } 9 & $A_{s t}$ & 36.0 & 30 & 40 & high & Stromal $\tau_{s t}$ target area \\
10 & $\lambda_{A_{s t}}$ & 3.0 & 0.5 & 14.0 & medium & Stromal $\tau_{s t}$ area energy weight \\
11 & $\phi_{s t}$ & 4.0 & 2.0 & 12.0 & high & Stromal $\tau_{s t}$ target isoperimetric quotient \\
12 & $\lambda_{\phi_{s t}}$ & 1.0 & 0.5 & 14.0 & medium & Stromal $\tau_{s t}$ IPQ energy weight \\
\hline Mechanism: Cell activation and growth \\
\hline 13 & $\varphi$ & 1.0 & 0.2 & 2.0 & medium & $\tau_{s}$ growth rate \\
14 & $\alpha_{s}$ & $4 \times 10^{-3}$ & $10^{-3}$ & $10^{-2}$ & medium & $\tau_{s}$ VegF activation level \\
15 & $\epsilon_{s}$ & 1.0 & 0.1 & 1.0 & high & $\tau_{s}$ VegF internal time constant \\
\hline
\end{tabular}

TABLE 3

Parameters 16 through 40 Governing the Model

\begin{tabular}{|c|c|c|c|c|c|c|}
\hline \multicolumn{7}{|c|}{ Mechanism: Differential adhesion } \\
\hline 16 & $J_{t, m}$ & 10 & 1 & 32 & high & Adhesion between $\tau_{t}$ and medium \\
\hline 17 & $J_{s, m}$ & 15 & 1 & 32 & high & Adhesion between $\tau_{s}$ and medium \\
\hline 18 & $J_{s t, m}$ & 16 & 1 & 32 & high & Adhesion between $\tau_{s t}$ and medium \\
\hline 19 & $J_{t, t}$ & 1 & 1 & 32 & high & Adhesion between $\tau_{t}$ and $\tau_{t}$ \\
\hline 20 & $J_{t, s}$ & 5 & 1 & 32 & high & Adhesion between $\tau_{t}$ and $\tau_{s}$ \\
\hline 21 & $J_{s, s}$ & 5 & 1 & 32 & high & Adhesion between $\tau_{s}$ and $\tau_{s}$ \\
\hline 22 & $J_{t, e}$ & 15 & 1 & 32 & high & Adhesion between $\tau_{t}$ and $\tau_{e}$ \\
\hline 23 & $J_{s, e}$ & 32 & 1 & 32 & high & Adhesion between $\tau_{s}$ and $\tau_{e}$ \\
\hline 24 & $J_{s, s t}$ & 10 & 1 & 32 & high & Adhesion between $\tau_{s}$ and $\tau_{s t}$ \\
\hline 25 & $J_{e, s t}$ & 15 & 1 & 32 & high & Adhesion between $\tau_{e}$ and $\tau_{s t}$ \\
\hline 26 & $J_{t, s t}$ & 16 & 1 & 32 & high & Adhesion between $\tau_{t}$ and $\tau_{s t}$ \\
\hline 27 & $J_{s t, s t}$ & 16 & 0.2 & 2.0 & high & Adhesion between $\tau_{s t}$ and $\tau_{s t}$ \\
\hline \multicolumn{7}{|c|}{ ECM degradation } \\
\hline 28 & $k_{u_{t}}$ & 0.008 & 0.2 & 2.0 & medium & ECM degradation when under $\tau_{t}$ \\
\hline 29 & $k_{n_{t}}$ & 0.008 & 0.2 & 2.0 & medium & ECM degradation when adjacent to $\tau_{t}$ \\
\hline 30 & $\eta$ & 0.25 & 0.2 & 2.0 & medium & ECM to fluid threshold \\
\hline 31 & $p_{e}$ & 0.30 & 0.2 & 2.0 & medium & ECM initial stromal fiber density \\
\hline \multicolumn{7}{|c|}{ Haptotaxis and chemotaxis } \\
\hline 32 & $\mu_{c}$ & $10^{5}$ & $5 \times 10^{4}$ & $2 \times 10^{5}$ & medium & VegF chemotactic force on $\tau_{t}$ \\
\hline 33 & $\mu_{h}$ & 100 & 0 & 200 & medium & ECM haptotactic force on $\tau_{t}$ \\
\hline \multicolumn{7}{|c|}{ Filopodia } \\
\hline 342 & $T_{f}$ & 200 & 0 & 300 & medium & Average duration of filopodia \\
\hline 35 & $l_{f}$ & 50 & 0 & 100 & low & Target length of filopodia \\
\hline 36 & $\lambda_{f}$ & 2.0 & 0.5 & 10.0 & medium & Length energy weight \\
\hline \multicolumn{7}{|c|}{ VegF secretion and uptake } \\
\hline 37 & $\lambda_{V}$ & $10^{-7}$ & $10^{-6}$ & $10^{-8}$ & low & VegF degradation rate \\
\hline 38 & $D_{V}$ & 0.048 & 0.01 & 0.1 & low & VegF diffusion rate \\
\hline 39 & $\beta_{V}$ & $1.5 \times 10^{-4}$ & $10^{-5}$ & $3.0 \times 10^{-4}$ & low & VegF binding and absorbtion rate \\
\hline 40 & $\gamma$ & 1.0 & 1.0 & 0.1 & low & VegF binding efficiency \\
\hline
\end{tabular}

constraint to the overall energy is controlled by a weight parameter $\lambda_{i}$, so the modeled shape of a cell of type $i$ is described by four parameters: two shape parameter targets $\left(A_{i}, \phi_{i}\right)$ and two relative energy weights $\lambda_{A_{i}}$ and $\lambda_{\phi_{i}}$.

Due to the importance of cell shape to morphology, all 12 shape parameters in Table 2 are considered for disruption by the search engine. During each simulation run, for those cell types that do not grow or divide (tumor, stromal, and tip) the shape targets and weights are fixed. For endothelial stalk cells, which grow when activated, the area target increases as described below. 
Since healthy cells do not fragment [63], the basic implementation of the GGH [50] is extended in this work to impose a continuity constraint on each cell's set of lattice sites.

\subsubsection{Endothelial Cell Activation and Growth}

During angiogenesis, endothelial cells in the existing vessel begin to grow when VegF cell surface receptors are activated. This is implemented indirectly in the model by having endothelial cells take up VegF from the extracellular medium and commence growing when the absorbed $\mathrm{VegF}$ exceeds a threshold [7]. Three search parameters control activation by VegF: $\varphi$ the growth rate of active endothelial cells; $\alpha_{t}$, the internal VegF threshold when endothelial cells begin to grow; and $\epsilon_{t}$, the accumulation rate of VegF within each endothelial cell. At each simulation iteration (MCS), the target area of a growing cell is incremented by $\varphi$, the growth rate parameter, which is bound from above by known doubling rates of endothelial cells [43]. When a growing cell's current area reaches twice its original target area, it is split into two equal daughter cells along its shortest axis. The parameters controlling activation and growth are given in Table 2 .

\subsubsection{Differential Adhesion}

Differential adhesion between contacting cells or between cells and the ECM is an important morphogenetic mechanism [63] that is used extensively in angiogenesis. The GGH model represents adhesion as an energy term that sums over all cell/cell, cell/ECM, and cell/medium borders in the lattice domain. Differential adhesion is implemented by weighting each border length based on the two object types involved, $\tau_{i}$ and $\tau_{j}$, and using parameter $J_{\tau_{i}, \tau_{j}}$, where $J$ values are inversely related to affinity (see [24]). In the angiogenesis simulation, six distinct types of objects are included and listed in Table 1. The search engine considers the effects of changing adhesion between any pair (listed in Table 3) of the six object types: endothelial tip cell, endothelial stalk cell, a stromal cell, a tumor cell, the ECM, and the fluid medium.

\subsubsection{Endothelial Tip Cell ECM Degradation}

The secretion of proteolytic enzymes such as matrix metallopoteinases by endothelial tip cells degrades the ECM proteins when the endothelial cells are near or in contact with the ECM [61]. In angiogenesis, this process is responsible for both the initial degradation of the basal lamina that allows a new sprout to extend from the existing vessel, and is an important factor in controlling the direction of sprout elongation. Without interactions between the extending stalk and the ECM, few anastomosing loops form [8]. In this model, proteolysis linearly decays the ECM by $k_{n_{t}}$ whenever the ECM fiber is next to an endothelial tip cell, and by $k_{u_{t}}$ whenever the ECM is under an endothelial cell (although the model is 2D, the amount of ECM is represented as an additional continuous layer, under the tissue layer). As the ECM is degraded, it no longer presents a barrier to cell movement. This is represented by the threshold parameter $\eta$. The initial density of the ECM in the stroma is controlled by the parameter $p_{e}$, the probability of a lattice site being occupied by ECM protein. Since ECM/endothelial cell interactions are significant in cancer development and treatment, the four model parameters $k_{u_{t}}, k_{n_{t}}, \eta$, and $p_{e}$ defined in Table 3, are considered by the search engine.

\subsubsection{Endothelial Haptotaxis and Chemotaxis}

In angiogenesis, the endothelial tip cell migrates by haptotaxis [7] based on the local concentration of ECM in the tissue, and by chemotaxis [36] based on the local concentration of VegF. To integrate these mechanisms into the GGH, two additional terms are added to the energy change function to favor cell boundary changes that move up the local ECM and the VegF gradients. The strength of the haptotactic force is controlled by parameter $k_{H}$ and the strength of the chemotactic force is controlled by parameter $\mu_{\sigma}$. Both these parameters are given in Table 3 and considered by the search engine.

\subsubsection{Endothelial Tip Filopodia}

Filopodia emanate from the endothelial tip cells and enable tip cells to explore their neighborhood to find endothelial cells from distinct sprouts, and appear to be critical in the rapid formation of vessel loops [22]. Filopodia influence haptotaxis as they guide movement along ECM fibers, and through chemotaxis guide the tip cell up the VegF gradient. Implicit modeling of filopodia as a pseudopod [48], [49] captures the general agitation of cell boundaries, but does not accurately capture the role of filopodia in tip cell exploration as these cell processes have been observed to reach two cell diameters from the cell center [28]. In this work, filopodia are modeled explicitly as contiguous protrusions from the surface of the cell that have duration and extent. Three parameters control the filopodia of the tip cells: the average duration (in MCS) $T_{f}$ of an individual filopodium protrusion, and two shape parameters implemented as an energy term with a target extension length $l_{f}$ and its $\lambda_{f}$ weight, given in Table 3. This explicit modeling of filopodia allows the search engine to explore novel and potentially important therapeutic interventions based on altering filopodial activity.

\subsubsection{Secretion and Uptake of VegF}

The recruitment of vessels by a tumor is accomplished through the endothelial cells' response to $\mathrm{VegF}$ and other secreted signal molecules. The secretion of VegF by tumor cells and its uptake by endothelial cells form a diffusion gradient between the tumor and the parent vessel. The endothelial tip cell is activated by $\mathrm{VegF}$ and begins chemotaxis toward the tumor. In the model, the level of VegF over the lattice sites in the tissue is controlled by a diffusion equation with four parameters, given in Table 3: the diffusion coefficient $D_{V}$, the degradation rate $\lambda_{V}>0$, the binding and uptake rate limit of $\beta$ over the endothelial cells, and finally $\gamma$, a scalar that represents the efficiency of $\mathrm{VegF}$ internal signalling transduction pathways. Altering $\mathrm{VegF}$ activity is a current clinical approach to reducing tumor growth by preventing vascularization [21], so all five VegF-related parameters are considered for disruption by the search engine.

\subsection{Oxygen Flow from Vessels to Tumor}

The final component of the simulation is the estimation of $\mathrm{O}_{2}$ delivered to the tumor during the formation of the network of new vessels. Computational studies [46], [45] have shown that the spatial structure and organization of the vessel network significantly influences blood flow and 


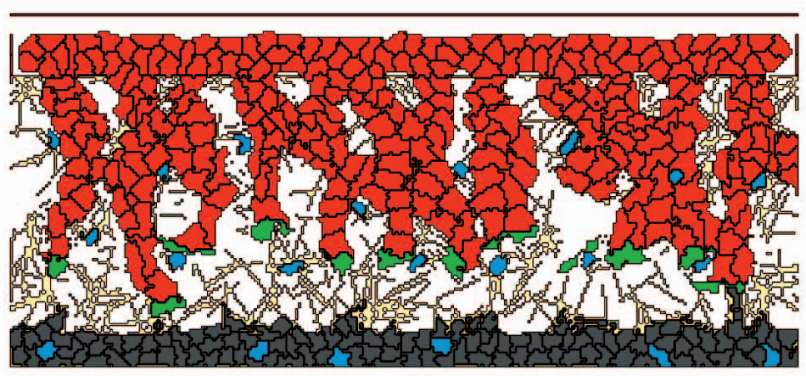

(a)

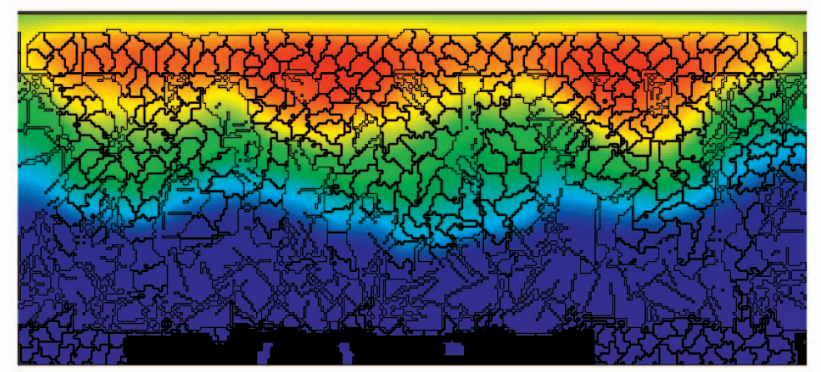

(b)

Fig. 2. (a) A typical vessel morphology produced by the simulation system after 600 MCS or 30 hours. (b) $O_{2}$ values over the domain for this morphology, where high concentrations of oxygen are red and low concentrations are blue to black. $O_{2}$ is secreted from vessels that form loops, diffuses through the stroma, and is absorbed by the tumor cells. The tissue array is 268 by 120 pixels. (a) Typical morphology. (b) Oxygen concentrations.

the consequent efficiency of oxygen delivery. To evaluate a given tissue morphology, a network-level layer is added to the model that extracts a network of pipes from contiguous lines of endothelial cells in the GGH lattice using techniques from image processing [39]. To compute the blood flow through each pipe, the method based on solving a set of Poiseuilles equations is used (first described in [2]). Once the blood flow through each vessel is determined, the diffusion of $\mathrm{O}_{2}$ through the tissue domain is computed, with the secretion of $\mathrm{O}_{2}$ from endothelial cells in each connected vessel proportional to blood flow in that vessel (see [14], [45], and [2] for more details). An example showing oxygen concentration over the tissue computed from a vessel morphology is given in Fig. 2. Finally, the $\mathrm{O}_{2}$ available for absorbtion by the tumor each 100 MCS of simulation time is accumulated and returned as the objective score. Diffusion constants of $\mathrm{O}_{2}$ were obtained from work in [42]. Because $\mathrm{O}_{2}$ is critical in all tissues and local disruption of $\mathrm{O}_{2}$ delivery would be a difficult therapeutic intervention, the oxygen delivery parameters were fixed and not explored by the search engine.

\section{Results}

To evaluate the effectiveness of the iterative improving beam search method, an empirical study was conducted utilizing parallel resources provided by the Arctic Region Supercomputing Center and the NSF TeraGrid through the Texas Advanced Computing Center. The study involved over 17,000 individual runs of the angiogenesis model, requiring over 120 days of total computation time, if run in serial.

The empirical study considered two principle issues: 1) the effectiveness of the search method in discovering high quality and novel solutions quickly and 2) an analysis of the discovered solutions as potential cancer therapies to see if the method "rediscovers" existing treatments and whether the method identifies novel and counter-intuitive solutions that have potential as future effective therapies.

\subsection{Search Method Effectiveness}

The performance of the improving beam search was evaluated by three experiments. First, the effectiveness of the ImprovingLocalBeamSearch method was evaluated by considering how the quality of the pool of near-Pareto optimal solutions improves as the algorithm is executed.
Second, the effectiveness of the ImprovingHillClimber method is evaluated by plotting the trajectory of nearPareto optimal solutions on the objective space. Third, the rate of discovery of near-Pareto optimal solutions is considered as the algorithm is run repeatedly.

\subsubsection{ImprovingLocalBeamSearch Effectiveness}

The results of this study are given in Fig. 3 and show the trajectory of the solution set frontier over the objective space (treatment cost vis. $\mathrm{O}_{2}$ supplied to the tumor) for reducing $k$, the numbers of active parameters, from three to one. To produce the graphs, the algorithm given in Table 1 was run 10 times. Earlier studies had considered the sensitivity of the approach to the size of population, but for populations over 64, the observed effect was minimal since the interaction among individuals is limited to competition for inclusion in the $\epsilon$-Pareto set. In studies reported here, the population size was fixed at 128 to maximize available parallel resources.

The effectiveness of the ImprovingLocalBeamSearch method in finding solutions that reduce both the solution cost and tumor $\mathrm{O}_{2}$ is demonstrated by the significant movement of the frontier toward the optimal point, and the difference in quality between the solution set found using pure Monte Carlo sampling to those in the final pooled set. Reviewing the results, it is clear that the Monte Carlo improving search is capable of quickly discovering novel and potentially important cancer therapies. All the solutions presented reduce the oxygen to the tumor, with many of the optimal solutions reducing oxygen by approximately 90 percent and some more expensive therapies eliminating $\mathrm{O}_{2}$ all together. This level of reduction would be expected to significantly slow the growth of the tumor and has the potential to improve the survival rate of patients [55].

One important criteria for evaluation of the search method is its ability to identify novel solutions that are not currently in use or development. To evaluate this aspect of the method, the summary of current antiangiogenesis therapies provided in [56], [18], and [44] was used to classify solutions as existing (i.e., nonnovel) if they change only one of the following model parameters: $\phi_{s}$ the growth rate of endothelial stalk cells; $\alpha_{s}$ or $\epsilon_{s}$, the VegF activation level of endothelial stalk cells; $\mu_{h}$, the haptotactic force exerted by ECM gradients on endothelial tip cells; $k_{u_{t}}, k_{n_{t}}$, or $\eta$, the decay of ECM by proteolytic enzymes secreted by 


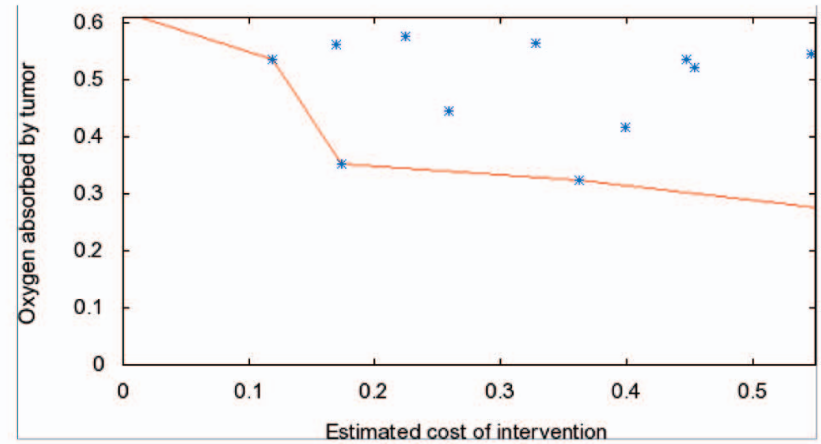

(a)

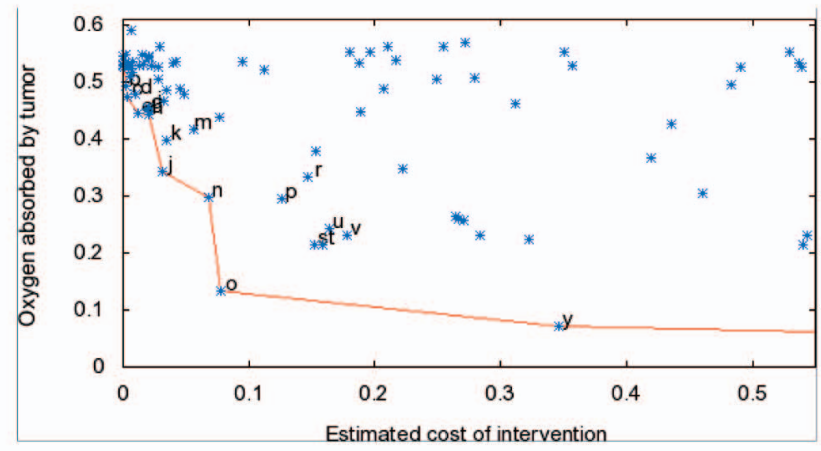

(c)

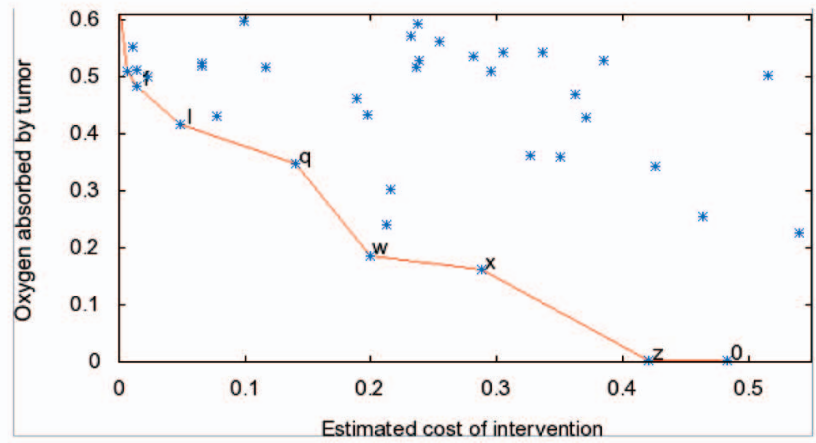

(b)

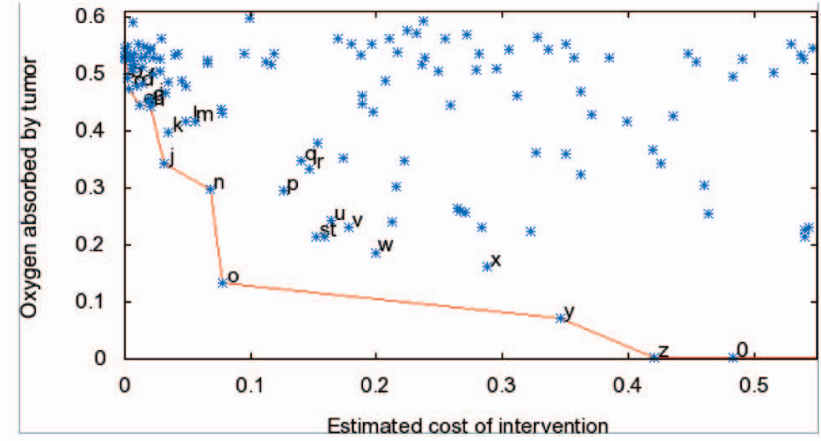

(d)

Fig. 3. Results: The Pareto optimal frontier (red line), solutions in $S_{k}$ (points) and near optimal potential cancer therapies (labeled points) identified by the improving local beam search process as $k$ is reduced from three to one. The best solutions provide the minimum $\mathrm{O}_{2}$ to the tumor (vertical axis) for the least estimated treatment cost (horizontal axis). Solution a (highest $\mathrm{O}_{2}$ solution on the vertical axis) represents the no treatment case at zero cost, but with the highest $\mathrm{O}_{2}$. Point labels correspond to the solutions shown in Fig. 7. (a) Solutions when $k=3$. (b) Solutions when $k=2$. (c) Solutions when $k=1$. (d) Complete solution pool $L^{*}$.

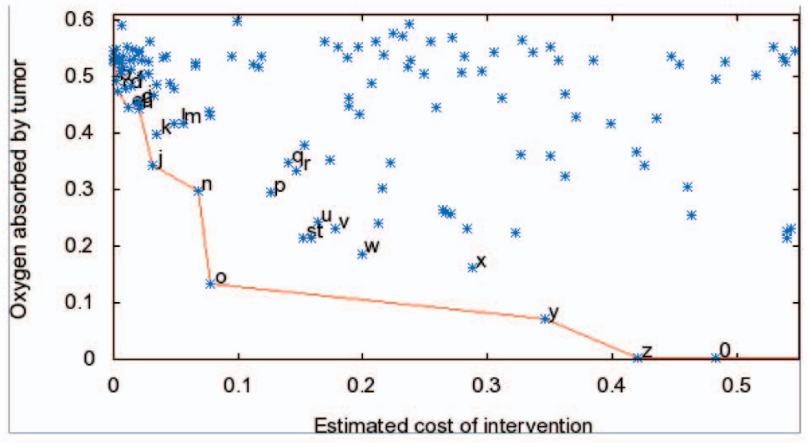

(a)

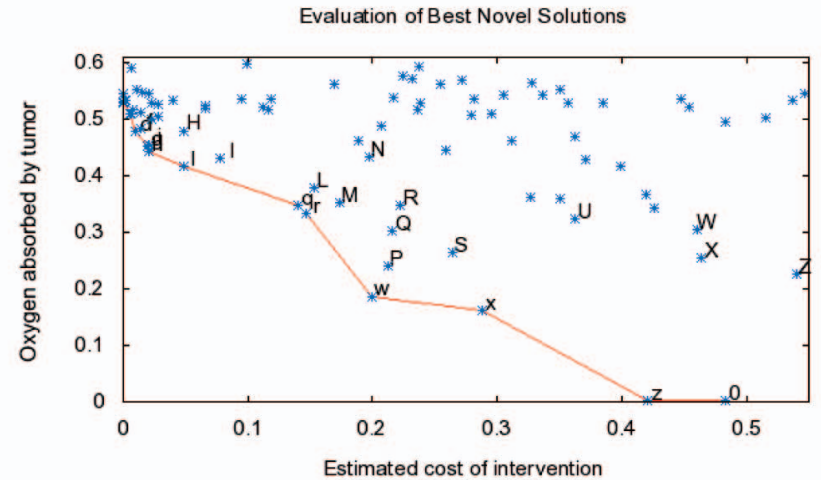

(b)

Fig. 4. Results: The best solutions and best novel solutions found by the improving beam search engine. The point labels correspond to the solutions shown in Fig. 7. (a) All solutions. (b) Only novel solutions.

endothelial tip cells; or $\lambda_{V}, D_{V}, \beta_{V}$, or $\gamma$, the secretion, diffusion, or uptake of VegF. Fig. 4 provides a summary of the best solutions and best novel solutions found by the improving beam search method. Note that many of the best solutions found in the near-Pareto optimal set represent novel solutions. Further analysis of the best solutions is provided in Section 4.2.

\subsubsection{ImprovingHillClimber Effectiveness}

The hill climber is intended to take the high-quality solutions found from the improving beam search engine and attempt to further improve the quality of each solution until a local minima is reached.
The results are given in Fig. 5 and show the trajectory for each best solution before and after being processed by the improving hill climber. For those solutions that show no arrow, the hill climber was unable to find a reduction in parameter deviations that reduced the tumor oxygen. The results show that the hill climber was able to find modest improvement in some of the solutions found by the improving beam search and significant improvement in a smaller number of solutions. The reason that some of the solutions could not be improved is likely due to the fixed step size used in reducing the parameter deviations. Future work is investigating how the use of adaptive and finer step sizes can identify maximal improvement in the solutions. 


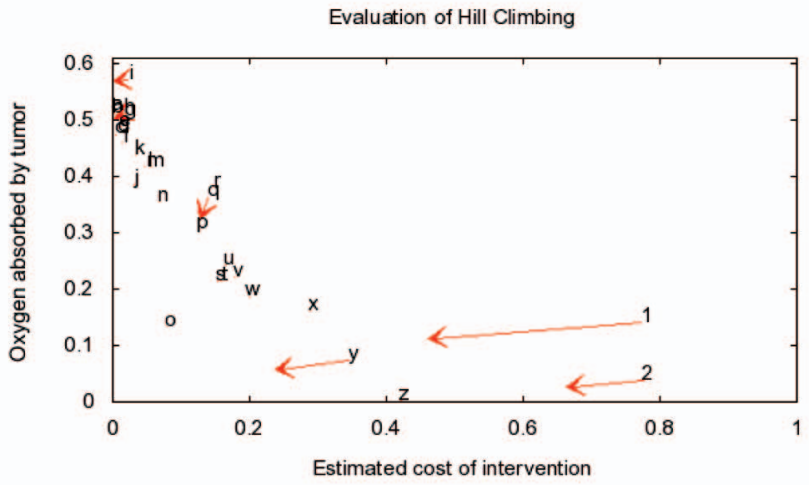

(a)

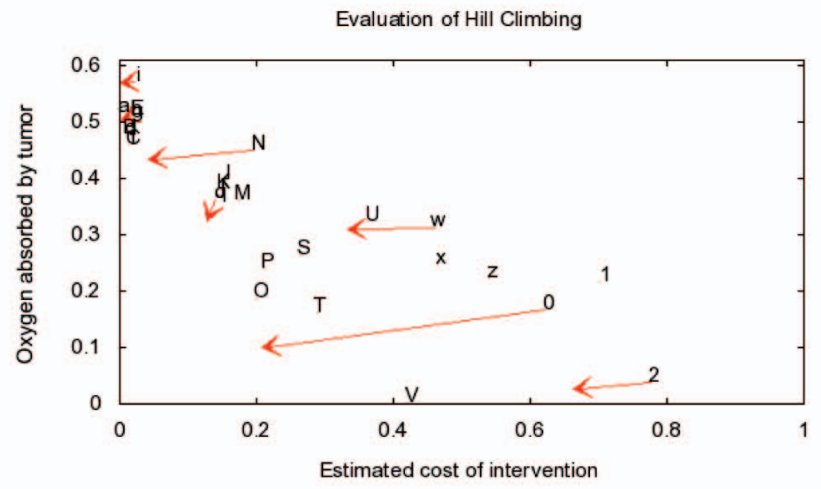

(b)

Fig. 5. Results: The effect of the hill climber on the best solutions found using the simplifying beam search. The point labels correspond to the solutions shown in Fig. 7. (a) All solutions. (b) Only novel solutions.

\subsubsection{Discovery Rate}

To investigate the rate of discovery of new solutions, the improving beam search (with a population size of 128) was run repeatedly and the best solutions pooled. After each beam-search run, the new best solutions found were compared to the existing pool of near-optimal solutions and the probability of discovering new solutions computed. A solution was considered new if the minimum distance between its parameter deviations and the parameter deviations of any of the pooled solutions was greater than 10 percent of the given parameter ranges. The results are given in Fig. 6.

The graphs in Fig. 6 show the discovery rate as a function of the number of solutions returned by the improving beam search. Each run of the improving bean search returned a batch of 60 of the highest quality solutions as defined below. The graphs show the results of running the improving beam search engine 10 times. The different lines on the graph correspond how the set of pooled solutions is defined. The lowest line gives the probability of discovery when only Pareto optimal solutions are retained in the solution pool, while the other lines give the probability of discovery when the solution pool is expanded to include near-Pareto optimal solutions. The quality of the nearPareto optimal solutions in the pool is controlled by the parameter $i$, which limits the number of dominating solutions each pooled solution can have. For $i=0$, only Pareto Optimal solutions are in the pool, for $i=20$, the pool contains all solutions that are dominated by no more than 20 other solutions in the pool. Fig. 6 a gives the discovery rate of all solutions, while Fig. $6 \mathrm{~b}$ gives the discovery rate of only those solutions that are novel (defined above).

The results show that the probability of a newly created solution being a member of the Pareto optimal set drops to almost 0 percent after 10 batch runs of the improving beam search. Considering the immense size of the combinatorial search space (with 40 real valued parameters), the improving beam search algorithm appears to rapidly find the highquality solutions that lie within the space.

\subsection{Solutions as Potential Cancer Therapies}

The second part of the empirical study analyzes the parameter deviations of the set of best and novel solutions found by the search engine and considers how the change in model parameters affect the resulting vessel morphologies and how these parameter changes maybe implemented as medical interventions in the future. To identify and organize the best solutions in $L *$, only those solutions dominated by no more than four other solutions were collected, then sorted by cost. The top 26 overall solutions and the top 26 novel solutions are illustrated in Fig. 7.

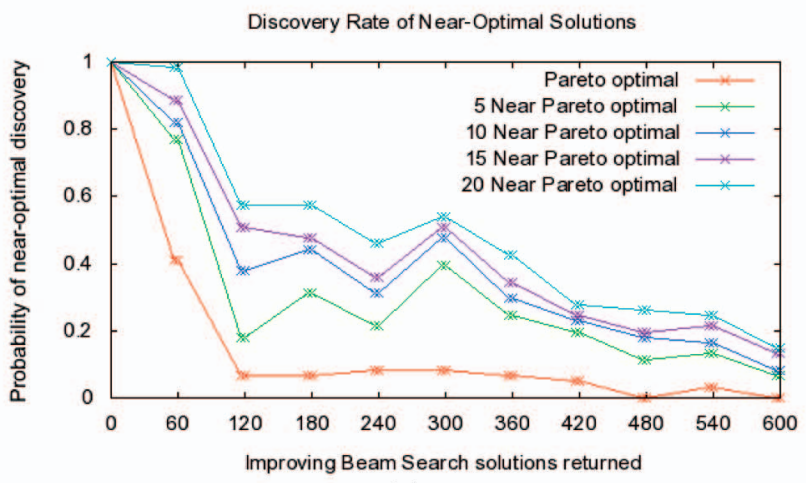

(a)

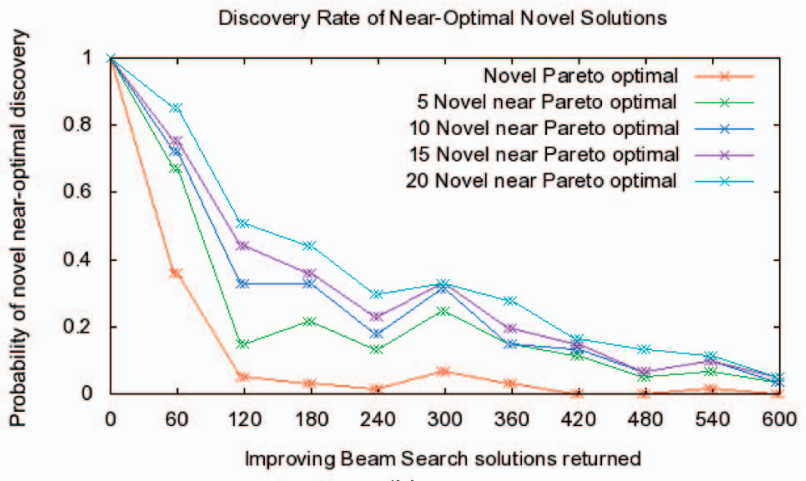

(b)

Fig. 6. Results: The rate of discovery for near-Pareto optimal solutions as the improving beam search engine is repeated and the solutions found are pooled. (a) shows all the solutions found, while (b) applies an additional filter that counts only novel solutions (see text for definition of novel). 


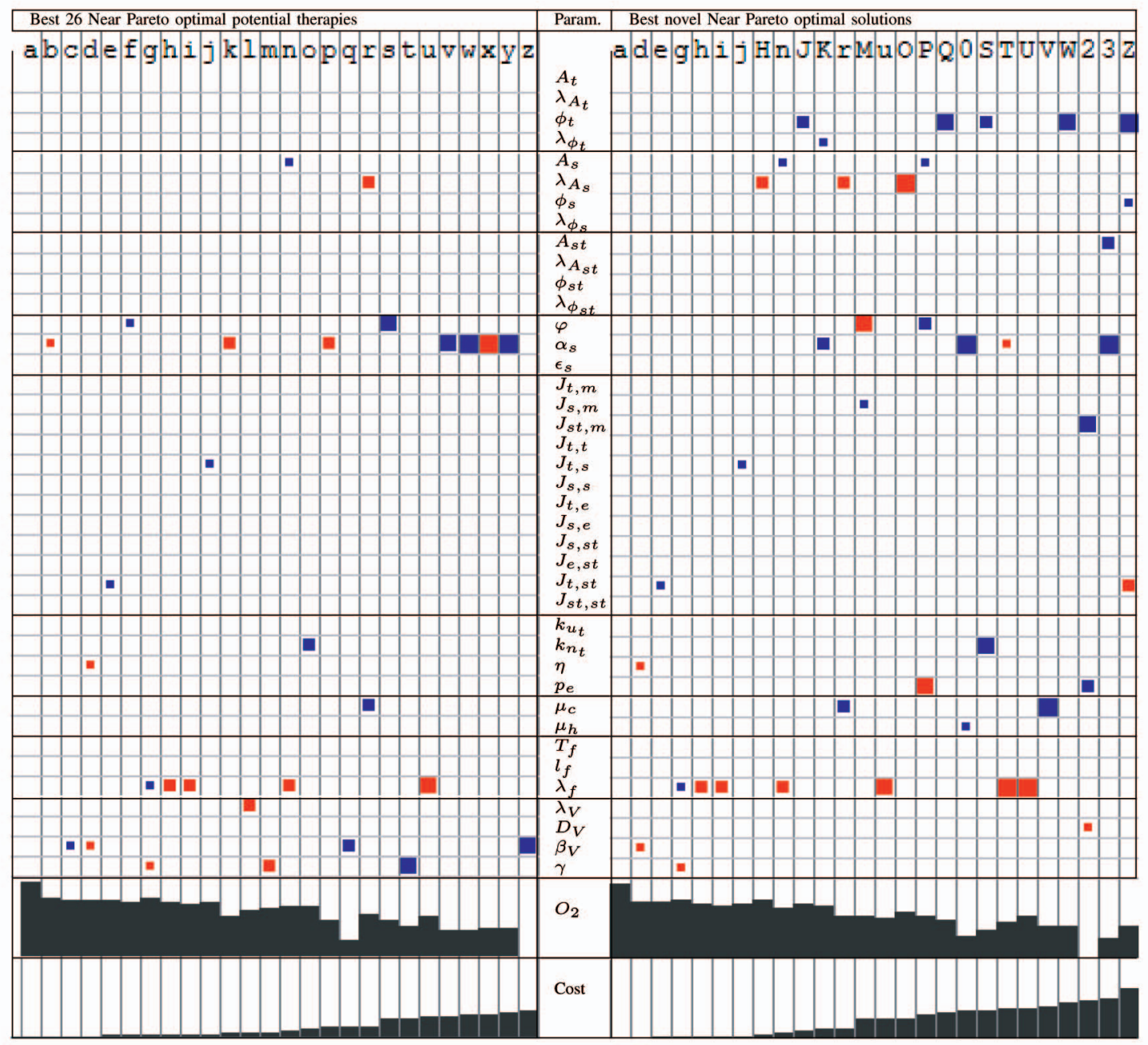

(a)

(b)

Fig. 7. Results: Near-Pareto optimal model disruptions found by the search engine. Each column represents one solution with the letter and number designations (across the top) corresponding to those in Figs. 3 and 4. The parameter disruptions for each solution is given in the column, with white meaning no change, red an increase in the parameter, and blue a decrease in the parameter. The parameters are summarized in Tables 2 and 3 and described in Section 3.3. Solution a represents the normative or no treatment case, with no parameter disruptions. The size of the red or blue box represents the magnitude of the change. The average $\mathrm{O}_{2}$ provided to the tumor and estimated cost is given in the last two rows. The solution set presented in (a) includes all the best solutions found. The solution set presented in (b) includes only the best novel solutions that explot neglected mechanisms or unexpected interactions among the multiple mechanisms.

Reassuringly, the best solution set includes many solutions that represent the rediscovery of treatments that are currently deployed or in development. Typical morphologies of a sample of best these solutions are given in Fig. 8. These four sample solutions are:

- Solution $\mathbf{f}$ reduces the growth rate of endothelial stalk cells $(\varphi)$ and thereby simulates the effects of various nonspecific chemotherapy strategies that aim to slow the growth of both tissue and tumor cells, or drugs that slow endothelial cell proliferation, such as Angiostatin ([44, Table 4]). A typical morphology for this solution is given in Fig. 8 and shows fewer loops leading to low oxygen delivery to the tumor.

- Solution 1 increases the decay rate of $\mathrm{VegF}$ in the tissue environment $\left(\lambda_{V}\right)$ so there is less $\mathrm{VegF}$ available to activate and guide the endothelial cells toward the tumor. This change in VegF reduces sprout growth and results in loops that lie close to the existing vessel (see Fig. 8). This solution corresponds to treatments using $\mathrm{VegF}$ antibodies, such as Avastin ([44, Table 2]), that bind and inactivate the defusing $\mathrm{VegF}$ in the tissue.

- Solution o reduces the effect of proteolytic enzymes secreted by endothelial tip cells in degrading nearby ECM $\left(k_{n_{t}}\right)$. This reduction in ECM degradation prevents the rapid expansion of new stalks through the tissue and leads to thin and fragmented stalks (see Fig. 8). This solution corresponds to treatments that block matrix metalloproteinases and other secreted proteases, such as BHPA or Batimastat ([44, Table 6]).

- Solution $\mathrm{q}$ reduces the uptake rate of $\operatorname{VegF}\left(\beta_{V}\right)$ by the endothelial stalk cells thereby leaving more VegF available in the environment and flattening the VegF gradient. The excess of $\mathrm{VegF}$ leads to increased endothelial stalk growth while the shallow VegF gradient slows the chemotactic movement of the 


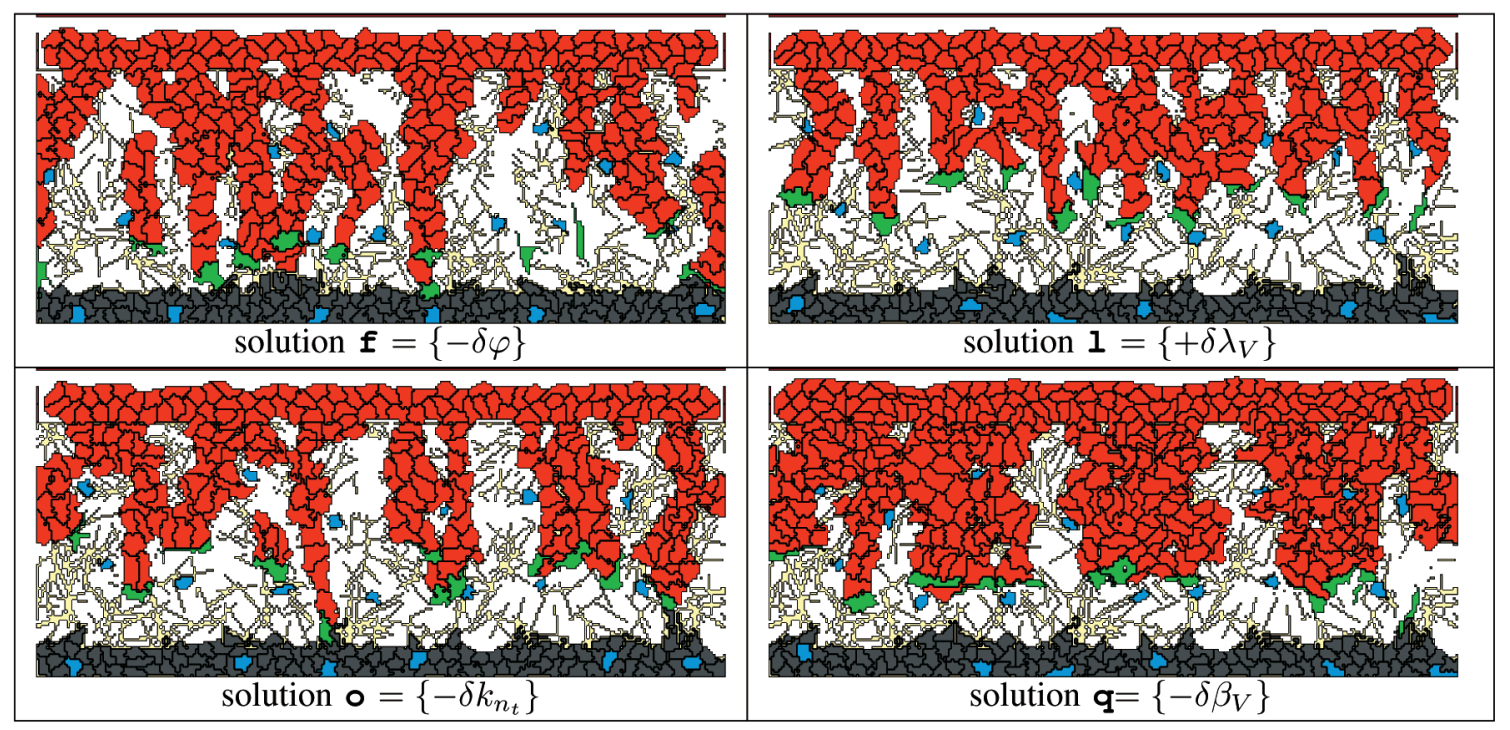

Fig. 8. Results: Typical morphologies for four selected treatments discovered by the search engine that correspond to treatments that are in use or trial. Solution labels are those given in Fig. 7.

endothelial tip cell toward the tumor, leading to clumps of stalk cells rather than distinct vessels. This solution corresponds to treatments that interfere with VegF receptors such as Angiozyme ([44, Table 3]).

The search engine discovers many novel solutions that disrupt mechanisms such as filopodia behavior, cell adhesion, or shape. Some of the more interesting solutions require the simultaneous alteration of two or three parameters to achieve the goal of reduced oxygen delivery to the tumor. It is unlikely that any of these solutions could be deduced from intuition. Four solutions that require single parameter alteration and two solutions that require multiple parameter alteration are presented below. Typical morphologies of a sample of novel solutions are given in Fig. 9. These six solutions are briefly discussed below:

- Solution e decreases the binding energy between endothelial tip cells and the stromal cells $\left(J_{t, s t}\right)$. Since $J$ is inversely related to adhesion, this decrease in $J_{t, s t}$ increases the adhesion between tip and stalk cells. In this case, many tip cells are "frozen" in contact with a stromal cell (Fig. 9). The increased stromal/tip affinity alters the path of tip cells and therefore the morphology of the trailing stalks. Vessels are straight and form few loops. While no anticancer drug currently exploits this mechanism, there are drugs under development that disrupt specific adhesive properties of activated endothelial cells through integrin receptors, such as LM609 ([44, Table 7]).

- Solution $\mathrm{U}$ increases the energy weight $\lambda_{f}$ parameter that enforces the length constraint of the filopodia extensions. This has the effect of reducing the mobility and excitation of the filopodia. This reduces the probability that growing sprouts merge to form a network of interconnected loops. Note that while the morphology illustrated in Fig. 9 exhibits some loops, they are close to the parent vessel and not connected laterally. No treatments that specifically target tip cell filopodia are currently available, but this solution does suggest testable treatments approaches that include the use of compounds that alter microfilament dynamics.

- Solution $\mathrm{H}$ increases the energy weight parameter $\lambda_{A_{s}}$ of the area of the endothelial stalk cells. Increasing this parameter creates more static stalk cells with reduced mobility. The apparent straightening of the sprouts maybe due to the "drag" the stalk cells impose upon the advancing tip cell. The straight sprouts don't encounter other sprouts and therefore do not form many loops.

- Solution $\mathbf{w}$ decreases the target IPQ parameter for endothelial tip cells $\left(\phi_{t}\right)$, causing tip cells to become more circular and explore less of their immediate environment. The effect is to reduce the probability that two nearby sprouts will contact and form a loop.

- Solution 0 sharply reduces oxygen delivery by simultaneously decreasing haptotaxis along the ECM $\left(\mu_{h}\right)$ and the threshold for endothelial cell activation $\left(\alpha_{s}\right)$. A typical morphology, shown in Fig. 9, show straight sprouts and many disconnected tip cells. Potential interventions suggested by this solution may include combination therapies with available agent Bevacizumab and an agent that reduces the interaction between tip cells and the ECM. Possibilities include antibodies against fibronectin or integrins, the primary fibronectin receptor, or pharmacological agents that block integrinmediated signaling.

- Solution 2 This solution reduces oxygen delivery to near zero and requires simultaneous changes in three parameters. In this case, the stomal cell/ medium binding energy $(J s t, m)$ and ECM fiber density $\left(p_{e}\right)$ are decreased, and the VegF diffusion rates $\left(D_{V}\right)$ are increased. Decreasing the Jst,m parameter has the effect of increasing the affinity 


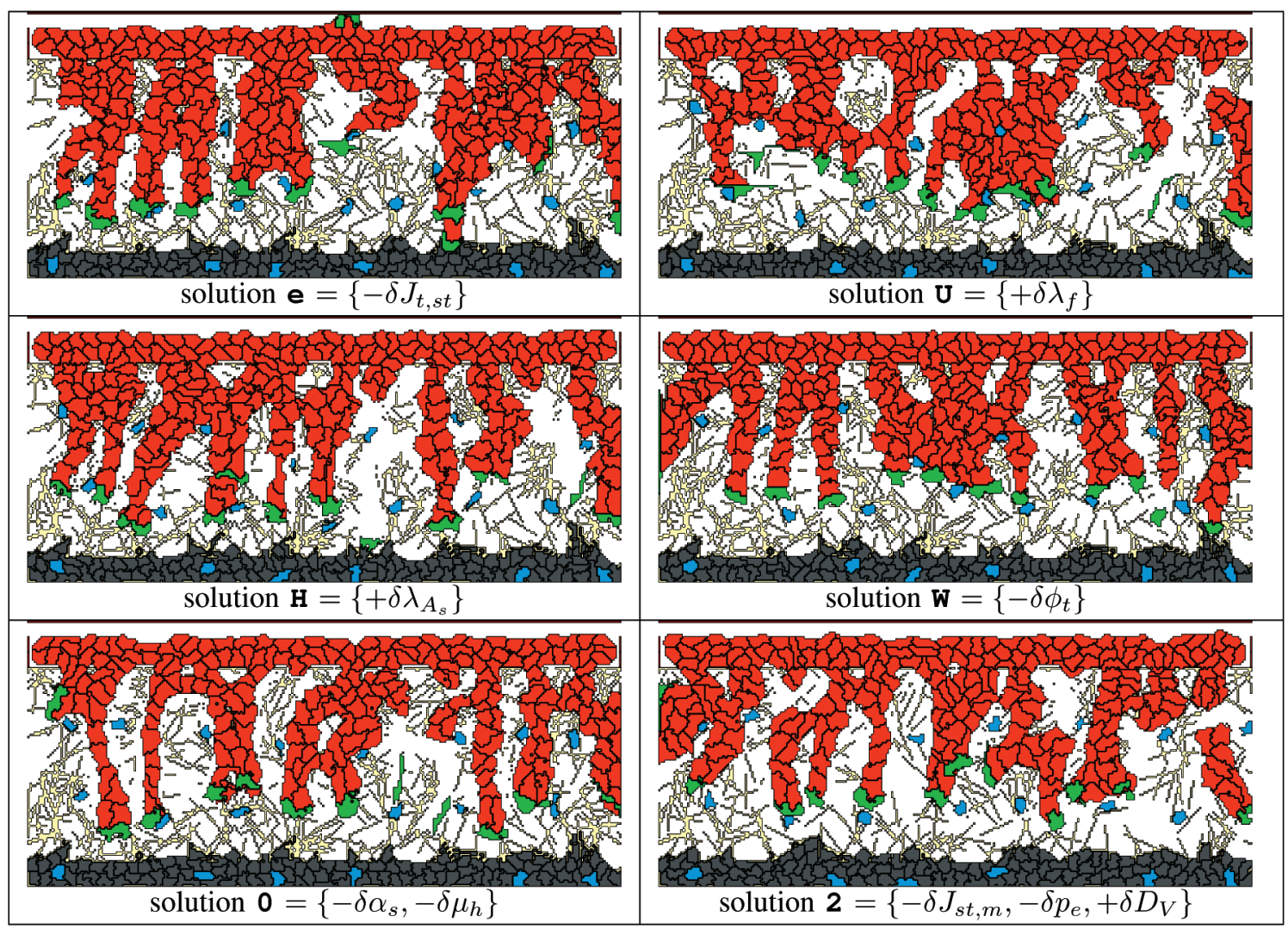

Fig. 9. Results: Typical morphologies for six selected novel potential treatments. Solution labels are those given in Fig. 7.

between stomal cells and medium. Increasing the VegF diffusion rate results in a shallower VegF gradient. This solution is more challenging to implement than any others previously discussed. The binding energy between stromal cells and medium is not a biologically relevant term, but it maybe possible to achieve the same result by reducing endothelial cell/stromal cell affinity through the blocking of integrin receptors. This would have the same effect on the morphology (see Fig. 9) where the stromal cells strongly prefer binding to medium over the endothelial cells. Similarly, there is no direct way to increase the VegF diffusion rate, but it maybe possible to consider ways to alter the steepness of the VegF concentration gradient. Of the three parameter modifications demanded by solution 2, changing the ECM fiber density is the most closely tied to biology. One possible approach would be targeted delivery of proteases such as matrix metalloproteinases to reduce ECM fiber density between a microtumor and the existing vasculature.

These novel solutions suggest possible parameter modifications that may not be achievable in the near term, but to discount these difficult solutions would miss the point of the search-based approach. The search provides nonintuitive and possibly effective treatments to consider, only some of which maybe realistic. Nonetheless, the process of reflecting on these solutions may lead to new, productive ways of thinking about blocking tumor-induced angiogenesis.

\section{Conclusions}

This report describes a biologically realistic model of angiogenesis in which complex tissue-level behaviors emerge from the interaction of cellular and subcellular properties. This angiogenesis model was used as an objective function for a parallel combinatorial search method for exploring the space of angiogenesis blocking interventions for treating cancer. The primary search proceeded using an improving Monte Carlo approach. Near-optimal solutions for blocking angiogenesis were found rapidly. These solutions were used as input for an improving Hill Climber search that discovered parameter values that most effectively block the delivery of oxygen to a solid tumor. In an empirical study that evaluated over 17,000 alternative solutions, many effective potential therapies were found.

In [44], existing clinical treatments or those close to deployment where grouped into five major classes based on the target mechanism of disruption (angiogenic factors, receptors of angiogenic factors, endothelial cell proliferation, metalloproteinases, and endothelial cell adhesion). The fact that the near-optimal set of solutions included examples from each of these five major classes strongly validates both the fidelity of the angiogenesis model and the search-based discovery approach, particularly, given the possibility that antiangiogenic treatments work through a combination of blocking new vessel growth to micro-tumors and renormalization of aberrant tumor-induced neovasculature. Moveover, many of the solutions discovered were novel and therefore not reported in [44], and some required the 
simultaneous disruption of two or three different model parameters that affect angiogenesis. The effective multiparameter solutions are particularly interesting because they cannot easily be derived by intuition. The results reported here suggest that this approach could help guide the development of new cancer treatments. Further refinements of the angiogenesis model to even more accurately reflect the known biology are likely to yield additional potential cancer treatment approaches for laboratory testing.

\section{ACKNOWLEDGMENTS}

This work was supported in part by a grant for high performance computing (HPC) resources from the Arctic Region Supercomputing Center at the University of Alaska Fairbanks as part of the Department of Defense High Performance Computing Modernization Program and by the National Science Foundation through TeraGrid resources provided by the Texas Advanced Computing Center.

\section{RefERENCES}

[1] M.A. Abo-Sinnaa and M.L. Husseinb, "An Algorithm for Generating Efficient Solutions of Multiobjective Dynamic Programming Problems," European J. Operational Research, vol. 80, no. 1, pp. 156-165, Jan. 1995.

[2] T. Alarcón, "A Cellular Automaton Model for Tumour Growth in Inhomogeneous Environment," J. Theoretical Biology, vol. 225, no. 2, pp. 257-274, Nov. 2003.

[3] F. Amyot, A. Small, and A.H. Gandjbakhche, "Stochastic Modeling of Tumor Induced Angiogenesis in a Heterogeneous Medium, the Extracellular Matrix," Proc. 28th Ann. IEEE Int'l Conf. Eng. in Medicine and Biology Soc. (EMBS '06) pp. 31463149, 2006.

[4] L. Arakelyan, V. Vainstein, and Z. Agur, "A Computer Algorithm Describing the Process of Vessel Formation and Maturation, and Its Use for Predicting the Effects of Anti-Angiogenic and AntiMaturation Therapy on Vascular Tumor Growth," Angiogenesis, vol. 5, no. 3, pp. 203-214, 2002.

[5] R. Auerbacha, R. Lewis, B. Shinners, L. Kubai, and N. Akhtar, "Angiogenesis Assays: A Critical Overview," Clinical Chemistry, vol. 49 , no. 1, pp. 32-40, 2003.

[6] S. Bandyopadhyay, S. Saha, U. Maulik, and K. Deb, "A Simulated Annealing-Based Multiobjective Optimization Algorithm: AMOSA," IEEE Trans. Evolutionary Computation, vol. 12, no. 3, pp. 269283, June 2008.

[7] A.L. Bauer, T.L. Jackson, and Y. Jiang, "A Cell-Based Model Exhibiting Branching and Anastomosis during Tumor-Induced Angiogenesis," Biophysical J., vol. 92, no. 9, pp. 3105-3121, May 2007.

[8] A.L. Bauer, T.L. Jackson, and Y. Jiang, "Topography of Extracellular Matrix Mediates Vascular Morphogenesis and Migration Speeds in Angiogenesis," PLoS Computational Biology, vol. 5, no. 7: e1000445, 2009, doi:10.1371/journal.pcbi.1000445.

[9] P. Boyle and B. Levin, eds., World Cancer Report 2008, IARC Nonserial, ISBN-13 9789283204237, ISBN-10 9283204239, 2008.

[10] H.M. Byrne, T. Alarcon, M.R. Owen, S.D. Webb, and P.K. Maini, "Modelling Aspects of Cancer Dynamics: A Review," Philosophical Transactions, Series A, Math., Physical, and Eng. Sciences, vol. 364, no. 1843, pp. 1563-1578, June 2006.

[11] H.M. Byrne, M.R. Owen, T.A. Alarcon, J. Murphy, and P.K. Maini, "Modelling the Response of Vascular Tumours to Chemotherapy: A Multiscale Approach," Math. Models and Methods in Applied Sciences, vol. 16, no. 7S, pp. 1219-1241, 2006.

[12] P. Carmeliet and R.K. Jain, "Angiogenesis in Cancer and Other Diseases," Nature, vol. 407, no. 6801, pp. 249-257, Sept. 2000.

[13] Carraway, "Generalized Dynamic Programming for Multicriteria Optimization," European J. Operational Research, vol. 44, no. 1, pp. $95-104,1990$.

[14] M.A.J.A. Chaplain, S.R.R. McDougall, and A.R.A.R. Anderson, "Mathematical Modeling of Tumor-Induced Angiogenesis," Ann. Rev. Biomedical Eng., vol. 8, pp. 233-257, Apr. 2006.
[15] A. Chattopadhyay and C. Seeley, "A Simulated Annealing Technique for Multiobjective Optimization of Intelligent Structures," Smart Materials and Structures, vol. 3, pp. 98-106, 1994.

[16] K. Deb, Multi-objective Optimization Using Evolutionary Algorithms. John Wiley and Sons, 2001.

[17] N. Ferrara, K. Hillan, and W. Novotny, "Bevacizumab (Avastin), A Humanized Anti-VEGF Monoclonal Antibody for Cancer Therapy," Biochemical and Biophysical Research Comm., vol. 333, no. 2, pp. 328-335, July. 2005.

[18] N. Ferrara and R.S. Kerbel, "Angiogenesis as a Therapeutic Target," Nature, vol. 438, no. 7070, pp. 967-974, Dec. 2005.

[19] R.S. Finley, "New Directions in the Treatment of Cancer: Inhibition of Signal Transduction," J. Pharmacy Practice, vol. 15, no. 1, pp. 5-16, Feb. 2002.

[20] C. Fonseca and P. Fleming, "An Overview of Evolutionary Algorithms in Multiobjective Optimization," Evolutionary Computation, vol. 3, no. 1, pp. 1-16, 1995.

[21] F.M. Gabhann and A.S. Popel, "Targeting Neuropilin-1 to Inhibit VEGF Signaling in Cancer: Comparison of Therapeutic Approaches," PLoS Computational Biology, vol. 2, no. 12: e180, Dec. 2006, doi:10.1371/journal.pcbi.0020180.

[22] H. Gerhardt, M. Golding, M. Fruttiger, C. Ruhrberg, A. Lundkvist, A. Abramsson, M. Jeltsch, C. Mitchell, K. Alitalo, D. Shima, and C. Betsholtz, "VEGF Guides Angiogenic Sprouting Utilizing Endothelial Tip Cell Filopodia," The J. Cell Biology, vol. 161, no. 6, pp. 1163-1177, June 2003.

[23] J.L. Gevertz and S. Torquato, "Modeling the Effects of Vasculature Evolution on Early Brain Tumor Growth," J. Theoretical Biology, vol. 243, no. 4, pp. 517-531, 2006.

[24] J.A. Glazier and F. Graner, "Simulation of the Differential Adhesion Driven Rearrangement of Biological Cells," Physical Rev. E, vol. 47, no. 3, pp. 2128-2154, 1993.

[25] S.L. Gupton and F.B. Gertler, "Filopodia: The Fingers that Do the Walking," Science Signaling, vol. 2007, no. 400, re5, Aug. 2007, doi: 10.1126/stke.4002007re5.

[26] J. Handl, D. Kell, and J. Knowles, "Multiobjective Optimization in Bioinformatics and Computational Biology," IEEE/ACM Trans. Computational Biology and Bioinformatics, vol. 4, no. 2, pp. 279-292, Apr. 2007.

[27] A.J. Hayes, L.Y. Li, and M.E. Lippman, "Science, Medicine, and the Future: Antivascular Therapy: A New Approach to Cancer Treatment," British Medical J., vol. 318, no. 7187, pp. 853-856, Mar. 1999.

[28] M. Hellstrom, L.-K. Phng, J.J. Hofmann, E. Wallgard, L. Coultas, P. Lindblom, J. Alva, A.-K. Nilsson, L. Karlsson, N. Gaiano, K. Yoon, J. Rossant, L.M. Iruela-Arispe, M. Kalén, H. Gerhardt, and C. Betsholtz, "Dll4 Signalling through Notch1 Regulates Formation of Tip Cells during Angiogenesis," Nature, vol. 445, pp. 776-780, Jan. 2007.

[29] P. Hogeweg, "Evolving Mechanisms of Morphogenesis: On the Interplay between Differential Adhesion and Cell Differentiation," J. Theoretical Biology, vol. 203, no. 4, pp. 317-333, Apr. 2000.

[30] M.T. Holderfield and C.C.W. Hughes, "Crosstalk between Vascular Endothelial Growth Factor, Notch, and Transforming Growth Factor-Beta in Vascular Morphogenesis," Circulation Research, vol. 102, pp. 637-652, Mar. 2008.

[31] J.A. Izaguirre, R. Chaturvedi, C. Huang, T. Cickovski, J. Coffland, G. Thomas, G. Forgacs, M. Alber, G. Hentschel, S.A. Newman, and J.A. Glazier, "Compucell, A Multi-Model Framework for Simulation of Morphogenesis," Bioinformatics, vol. 20, no. 7, pp. 1129-1137, May 2004.

[32] T. Jackson, "A Mathematical Model to Study the Effects of Drug Resistance and Vasculature on the Response of Solid Tumors to Chemotherapy," Math. Biosciences, vol. 164, no. 1, pp. 17-38, Mar. 2000.

[33] R.K. Jain, R.T. Tong, and L.L. Munn, "Effect of Vascular Normalization by Antiangiogenic Therapy on Interstitial Hypertension, Peritumor Edema, and Lymphatic Metastasis: Insights from a Mathematical Model," Cancer Research, vol. 67, no. 6, pp. 27292735, Mar. 2007

[34] A. Jemal, R. Siegel, E. Ward, Y. Hao, J. Xu, T. Murray, and M.J Thun, "Cancer Statistics, 2008," CA: A Cancer J. for Clinicians, vol. 58, no. 2, pp. 71-96, Mar. 2008.

[35] J.W. Ji, N.M. Tsoukias, D. Goldman, and A.S. Popel, "A Computational Model of Oxygen Transport in Skeletal Muscle for Sprouting and Splitting Modes of Angiogenesis," J. Theoretical Biology, vol. 241, no. 1, pp. 94-108, July 2006. 
[36] J. Kafer, P. Hogeweg, and A.F. Marée, "Moving Forward Moving Backward: Directional Sorting of Chemotactic Cells due to Size and Adhesion Differences," PLoS Computational Biology, vol. 2, no. 6: e56, June 2006, doi:10.1371/journal.pcbi.0020056.

[37] Y. Lan and G.A. Papoian, "The Stochastic Dynamics of Filopodial Growth," Biophysical J., vol. 94, no. 10, pp. 3839-3852, May 2008.

[38] W.B. Langdon and R. Poli, "Fitness Causes Bloat," Soft Computing in Engineering Design and Manufacturing, pp. 23-27, SpringerVerlag, 1997.

[39] T.-C. Lee, R.L. Kashyap, and C.-N. Chu, "Building Skeleton Models via 3D Medial Surface/Axis Thinning Algorithms," CVGIP: Grapical Models and Image Processing, vol. 56, no. 6, pp. 462-478, Nov. 1994.

[40] H. Levine, S. Pamuk, B. Sleeman, and M. Nilsen-Hamilton, "Mathematical Modeling of Capillary Formation and Development in Tumor Angiogenesis: Penetration into the Stroma," Bull. of Math. Biology, vol. 63, no. 5, pp. 801-863, 2001.

[41] M. James Ji and A. Popel, "Multi-Scale Computational Models of Pro-Angiogenic Treatments in Peripheral Arterial Disease," Annals of Biomedical Eng., vol. 35, no. 6, pp. 982-994, June 2007.

[42] J.D.B. Macdougall and M. Mccabe, "Diffusion Coefficient of Oxygen through Tissues," Nature, vol. 215, no. 5106, pp. 1173$1174,1967$.

[43] T. Maciag, G.A. Hoover, M.B. Stemerman, and R. Weinstein, "Serial Propagation of Human Endothelial Cells In Vitro," The J. Cell Biology, vol. 91, no. 2, pp. 420-426, Nov. 1981.

[44] J.L. Mauriz and J. González-Gallego, "Antiangiogenic Drugs: Current Knowledge and New Approaches to Cancer Therapy," J. Pharmaceutical Sciences, vol. 97, no. 10, pp. 4129-4154, 2008.

[45] S.R. McDougall, A.R. Anderson, and M.A. Chaplain, "Mathematical Modelling of Dynamic Adaptive Tumour-Induced Angiogenesis: Clinical Implications and Therapeutic Targeting Strategies," J. Theoretical Biology, vol. 241, no. 3, pp. 564-589, Aug. 2006.

[46] S.R. McDougall, A.R. Anderson, M.A. Chaplain, and J.A. Sherratt, "Mathematical Modelling of Flow through Vascular Networks: Implications for Tumour-Induced Angiogenesis and Chemotherapy Strategies," Bull. of Math Biology, vol. 64, no. 4, pp. 673-702, July 2002.

[47] R.M. Merks, S.V. Brodsky, M.S. Goligorksy, S.A. Newman, and J.A. Glazier, "Cell Elongation is Key to in silico Replication of In Vitro Vasculogenesis and Subsequent Remodeling," Developmental Biology, vol. 289, no. 1, pp. 44-54, Jan. 2006.

[48] R.M. Merks and J.A. Glazier, "A Cell-Centered Approach to Developmental Biology," Physica A, vol. 352, pp. 113-130, 2005.

[49] R.M. Merks, E.D. Perryn, A. Shirinifard, and J.A. Glazier, "Contact-Inhibited Chemotaxis in De Novo and Sprouting Blood-Vessel Growth," PLoS Computational Biology, vol. 4, no. 9: e1000163, 2008, doi:10.1371/journal.pcbi.1000163.

[50] R.M.H. Merks, S.A. Newman, and J.A. Glazier, "Cell-Oriented Modeling of In Vitro Capillary Development," Cellular Automata, pp. 425-434, Springer, 2004.

[51] T. Morisada, Y. Kubota, T. Urano, T. Suda, and Y. Oike, "Angiopoietins and Angiopoietin-Like Proteins in Angiogenesis," Endothelium, vol. 13, no. 2, pp. 71-79, Apr. 2006.

[52] S.M. Peirce, "Computational and Mathematical Modeling of Angiogenesis," Microcirculation, vol. 15, no. 8, pp. 739-751, 2008.

[53] C. Ruegg and N. Mutter, "Anti-Angiogenic Therapies in Cancer : Achievements and Open Questions," Bull. of Cancer, vol. 94, pp. 753-762, Sept. 2007.

[54] Shiba, Yuji, Takahashi, Masafumi, Ikeda, and Uichi, "Models for the Study of Angiogenesis," Current Pharmaceutical Design, vol. 14, no. 4, pp. 371-377, Feb. 2008.

[55] S. Shinkaruk, M. Bayle, G. Laïn, and G. Déléris, "Vascular Endothelial Cell Growth Factor (VEGF), an Emerging Target for Cancer Chemotherapy," Current Medicinal Chemistry-Anti-Cancer Agents, vol. 3, no. 2, pp. 95-117, Mar. 2003.

[56] F. Shojaei and N. Ferrara, "Antiangiogenic Therapy for Cancer: An Update," The Cancer J., vol. 13, no. 6, pp. 345-348, Nov. 2007.

[57] W.G. Stetler-Stevenson, "Matrix Metalloproteinases in Angiogenesis: A Moving Target for Therapeutic Intervention," The J. Clinical Investigation, vol. 103, no. 9, pp. 1237-1241, May 1999.

[58] S.F. Stewman and A.R. Dinner, "Lattice Model for Self-Assembly with Application to the Formation of Cytoskeletal-Like Structures," Physical Rev. E (Statistical, Nonlinear, and Soft Matter Physics), vol. 76, no. 1, p. 016103, July 2007, doi:10.1103/ PhysRevE.76.016103.
[59] B. Suman and P. Kumar, "A Survey of Simulated Annealing as a Tool for Single and Multiobjective Optimization," J. Operational Research Soc., vol. 57, no. 18, pp. 1143-1160, 2006.

[60] A. Suppapitnarm, K. Seffen, G. Parks, and P. Clarkson, "A Simulated Annealing Algorithm for Multiobjective Optimization," Eng. Optimization, vol. 33, no. 1, pp. 59-85, 2000.

[61] S. Turner and J.A. Sherratt, "Intercellular Adhesion and Cancer Invasion: A Discrete Simulation Using the Extended Potts Model," J. Theoretical Biology, vol. 216, no. 1, pp. 85-100, May 2002.

[62] I. Walker and H. Newell, "Do Molecularly Targeted Agents in Oncology Have Reduced Attrition Rates?" Nature Rev. Drug Discovery, vol. 8, no. 1, pp. 15-16, Nov. 2008.

[63] L. Wolpert, J. Smith, T. Jessell, and P. Lawrence, Principles of Development, third ed., Oxford Univ. Press, 2007.

[64] E. Zitzler, K. Deb, and L. Thiele, "Comparison of Multiobjective Evolutionary Algorithms: Empirical Results," Evolutionary Computation, vol. 8, no. 2, pp. 173-195, 2000.

[65] E. Zitzler and L. Thiele, "Multiobjective Evolutionary Algorithms: A Comparative Case Study and the Strength Pareto Approach," IEEE Trans. Evolutionary Computation, vol. 3, no. 4, pp. 257-271, Nov. 1999.

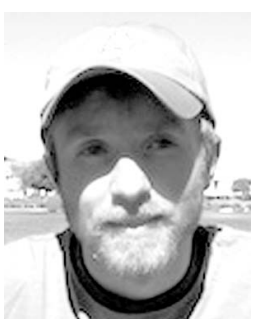

Arthur W. Mahoney received the BS degree in computer science and computational mathematics from Utah State University in 2009. He is a $\mathrm{PhD}$ student in the School of Computing at the University of Utah. He currently is performing research in magnetically controlled, wireless robotics under the mentorship of Dr. Jake Abbott. As an undergraduate, he conducted research in computational search methods for discovering novel cancer therapies, massively parallel graph search strategies, and robotic motion planning. He particularly enjoys sailing, wooden boats, and anything having to do with the outdoors.

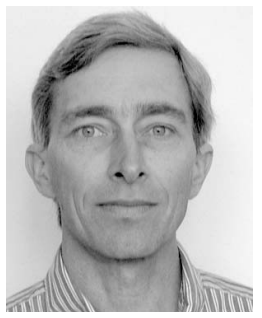

Gregory J. Podgorski received the PhD degree in cellular and molecular biology from Penn State University in 1983. He is an associate professor in the Biology Department of Utah State University. He held postdoctoral positions at Columbia University and the Max Planck Institute for Biochemistry. He conducts research into computational modeling of developmental processes and in instructional technology. $\mathrm{He}$ teaches majors and nonmajors general biology, genetics, and developmental biology, is a National Academies Teaching fellow, and has won teaching awards at Utah State University.

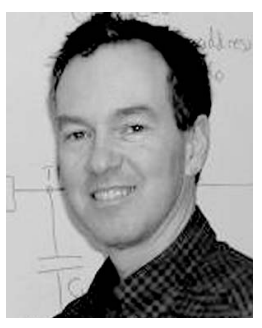

Nicholas S. Flann received the PhD degree in computer science from Oregon State University in 1991. He is an associate professor of computer science at Utah State University (USU). He is currently conducting research in multiscale models of tissue development, the evolution and modeling of genetic regulatory networks, and use of search engines and massive parallel computation to solve complex combinatorial optimization problems. He currently teaches classes in advanced algorithms, machine intelligence, and bioinformatics at USU. He currently serves as an associate editor for the Journal BioSystems.

$\triangleright$ For more information on this or any other computing topic, please visit our Digital Library at www.computer.org/publications/dlib. 